\title{
Machine learning using approximate inference Variational and sequential Monte Carlo methods
}

Christian Andersson Naesseth

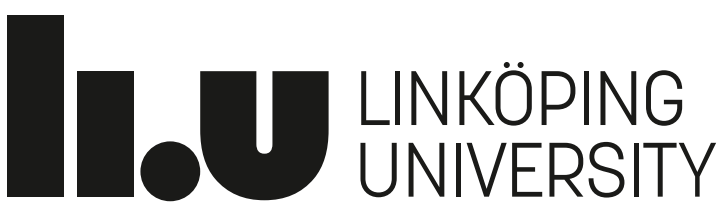


Cover illustration: The collection of particle ancestral paths in the fully adapted sequential Monte Carlo algorithm, with stratified resampling, applied to a linear Gaussian state space model.

Linköping studies in science and technology. Dissertations.

No. 1969

Machine learning using approximate inference: Variational and sequential Monte Carlo methods

Christian Andersson Naesseth

christian.a.naesseth@liu.se

www.control.isy.liu.se

Division of Automatic Control

Department of Electrical Engineering

Linköping University

SE-581 83 Linköping

Sweden

ISBN 978-91-7685-161-6

ISSN 0345-7524

Copyright $(0) 2018$ Christian Andersson Naesseth

Printed by LiU-Tryck, Linköping, Sweden 2018 
To my family 



\section{Abstract}

Automatic decision making and pattern recognition under uncertainty are difficult tasks that are ubiquitous in our everyday life. The systems we design, and technology we develop, requires us to coherently represent and work with uncertainty in data. Probabilistic models and probabilistic inference gives us a powerful framework for solving this problem. Using this framework, while enticing, results in difficult-to-compute integrals and probabilities when conditioning on the observed data. This means we have a need for approximate inference, methods that solves the problem approximately using a systematic approach. In this thesis we develop new methods for efficient approximate inference in probabilistic models.

There are generally two approaches to approximate inference, variational methods and Monte Carlo methods. In Monte Carlo methods we use a large number of random samples to approximate the integral of interest. With variational methods, on the other hand, we turn the integration problem into that of an optimization problem. We develop algorithms of both types and bridge the gap between them.

First, we present a self-contained tutorial to the popular sequential Monte Carlo (SMC) class of methods. Next, we propose new algorithms and applications based on SMC for approximate inference in probabilistic graphical models. We derive nested sequential Monte Carlo, a new algorithm particularly well suited for inference in a large class of high-dimensional probabilistic models. Then, inspired by similar ideas we derive interacting particle Markov chain Monte Carlo to make use of parallelization to speed up approximate inference for universal probabilistic programming languages. After that, we show how we can make use of the rejection sampling process when generating gamma distributed random variables to speed up variational inference. Finally, we bridge the gap between SMC and variational methods by developing variational sequential Monte Carlo, a new flexible family of variational approximations. 



\section{Populärvetenskaplig sammanfattning}

Fungerar den nya medicinen som vi har utvecklat? Vilket spel, film eller bok ska vi rekommendera härnäst? Bör jag investera nu eller bör jag vänta? Dessa är några exempel på frågor som man kan svara på med hjälp av maskininlärning (eng. machine learning). Maskininlärning handlar om metoder för att få en dator att automatiskt lära sig något från insamlad data. Data kan vara lite allt möjligt som man kan spara på en dator, alltifrån aktuell aktiekurs till vem man känner på Facebook. Datorn lär sig sen oftast en matematisk modell som beskriver datan. Med denna matematiska modell kan man: i) studera underliggande strukturer och mekanismer (t.ex. "hur påverkar tryck och temperatur vädret?"), ii) förutsäga hur framtida data kan se ut (t.ex. "kommer det regna imorgon?"), och iii) ta beslut (t.ex. "det är inte troligt att det regnar imorgon, så vi behöver inte ta med ett paraply").

Matematiska modeller används överallt inom teknologin och vetenskapens alla grenar. I många fall byggs modeller baserat på data som är insamlad under osäkra förhållanden eller med en i grunden slumpmässig variation. Dessa förhållanden lämpar sig väl för att jobba med sannolikheter, ett matematiskt koncept som blivit centralt i modern statistik och maskininlärning. Att lära sig och använda matematiska modeller baserat på sannolikhetsteori kallas för statistisk inferens. I många tillämpningar är datan vi samlat in för storskalig och modellen vi byggt för komplicerad för att exakt uträkningar ska vara möjliga. Detta betyder att vi måste använda och införa systematiska approximationer, vi utför approximativ inferens.

I denna avhandling studerar vi och utvecklar flera olika metoder för approximativ inferens med användning inom maskininlärning. Vi presenterar en introduktion till en populär klass av metoder som är speciellt användbara om ens matematiska modell beskriver data som varierar i tiden, till exempel vädret. Vi presenterar nya strategier som på flera sätt underlättar och effektiviserar den automatiska lärande-proccesen så att vi enklare kan hantera storskalig data och bättre, mer avancerade, modeller. 



\section{Acknowledgments}

These five+ years have been a truly enjoyable experience. I have had the pleasure of getting to know, and collaborate with, many wonderful people.

First of all, I would like to thank my amazing supervisors Fredrik Lindsten and Thomas Schön. Thank you both for your positive attitudes, support, and the energy you put into helping me during my graduate studies. Fredrik, with your depth of knowledge, attention to detail, and creativity, it has been a pleasure to have you as my supervisor. And Thomas, thanks for helping with the big picture, inspiration and coaching.

A good working environment is important. I would like to thank former head of division Svante Gunnarsson, current head of division Martin Enqvist, and Ninna Stensgård for their helpfulness and diligence in this respect. Thanks also go out to all my great current and former colleagues! Especially (in no particular order) Jonas Linder, Sina Khoshfetrat Pakazad, Hanna Nyqvist, Zoran Sjanic, Daniel Petersson, André Carvalho Bittencourt, Patrik Axelsson, Manon Kok, Martin Skoglund, Tohid Ardeshiri, Niklas Wahlström, Johan Dahlin, Clas Veibäck, Martin Lindfors, and everyone else; thank you all for everything from the entertaining fika-discussions and BBQs, to the enjoyable board game evenings. Thanks also to CADICS, a Linnaeus Center funded by the Swedish Research Council, for providing the funds for my position.

During my graduate studies I have had the privilege to be able to go for several research visits. Thank you Frank Wood for inviting me to visit your amazing research group at Oxford University. During this visit, I had a great time working with and getting to know Tom Rainforth (thanks also for letting me use your spare room!), Brooks Paige, and Jan-Willem van de Meent. Thanks David Blei for letting me spend time as a visiting student researcher in your awesome group at Columbia University. While there I had the pleasure of collaborating with and getting to know Francisco Ruiz, Scott Linderman, Rajesh Ranganath, and Alp Kucukelbir. Thanks also to Francisco and Maja for saving the world together from certain doom! Another big thanks to Sebastian Nowozin for inviting me to Microsoft Research Cambridge for the summer. Working together with you, Sebastian Tschiatschek, and Jan Stuehmer was a great experience.

Thank you to my proofreaders Martin Lindfors, Andreas Svensson, Carl Andersson, Jalil Taghia, Fredrik Lindsten, and Thomas Schön. While I have tried my best to keep the thesis as error-free as possible, all remaining errors are entirely my own.

A big thanks go out to my family of course. Thanks to my parents, Elizabeth Naesseth and Ingemar Andersson, and siblings, Malin Fridh, Daniélle Andersson Bredenberg and Andree Andersson Bredenberg, for your support and encouragement; I love you all. I especially want to thank my mom, for always being there for me and for all the love you have always given me. 
Last but not least, thank you to my wonderful wife Ziwei Naesseth $\mathrm{Hu}$, for existing, for your support, and for being awsome :) Love you more than I can express!

Linköping, November 2018 Christian Andersson Naesseth 


\section{Background}

1 Introduction 3

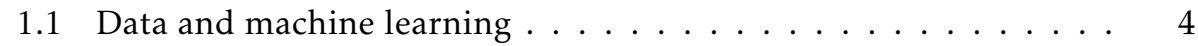

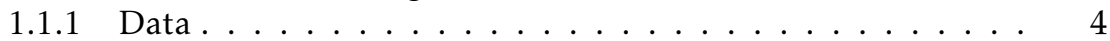

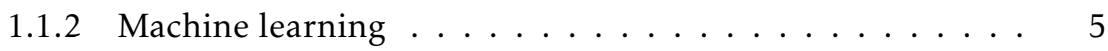

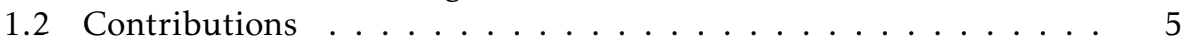

1.2.1 Elements of sequential Monte Carlo . . . . . . . . . . 6

1.2.2 Sequential Monte Carlo for graphical models . . . . . . . 6

1.2.3 Nested sequential Monte Carlo . . . . . . . . . . . . . 7

1.2.4 Variational Monte Carlo . . . . . . . . . . . . . . . 8

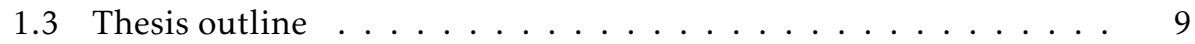

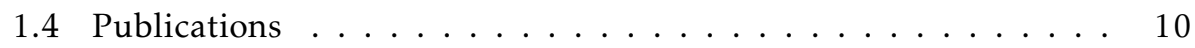

2 Probabilistic machine learning 13

2.1 Modeling . . . . . . . . . . . . . . . . . . . . 14

2.2 Inference . . . . . . . . . . . . . . . . . 16

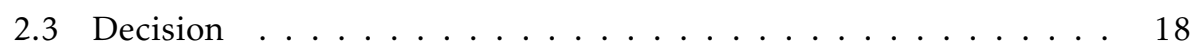

3 Approximate inference 21

3.1 Monte Carlo methods . . . . . . . . . . . . . . . . . . 22

3.1.1 The Monte Carlo idea . . . . . . . . . . . . . . . . . . 22

3.1 .2 Rejection sampling . . . . . . . . . . . . . 23

3.2 Variational inference . . . . . . . . . . . . . . . . 25

3.2 .1 The variational idea . . . . . . . . . . . . . . 26

3.2.2 Coordinate ascent variational inference . . . . . . . 27

3.2.3 Stochastic gradient variational inference . . . . . . . . . 28

3.2.4 Variational expectation-maximization $\ldots \ldots \ldots . \ldots 30$

4 Concluding remarks $\quad 33$

Bibliography $\quad 35$ 


\section{Publications}

A Elements of Sequential Monte Carlo 43

1 Introduction . . . . . . . . . . . . . . . . 45

1.1 Historical Background . . . . . . . . . . . . . 46

1.2 Probabilistic Models and Target Distributions . . . . . . . . 46

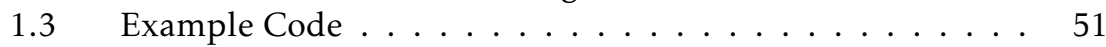

1.4 Outline ....................... 51

2 Importance Sampling to Sequential Monte Carlo . . . . . . . . . . 52

2.1 Importance Sampling . . . . . . . . . . . . . . . 52

2.2 Sequential Monte Carlo . . . . . . . . . . . . . 57

2.3 Analysis and Convergence . . . . . . . . . . . . . 64

3 Learning Proposals and Twisting Targets . . . . . . . . . . . . 67

3.1 Designing the Proposal Distribution . . . . . . . . . 67

3.2 Adapting the Target Distribution . . . . . . . . 78

4 Discussion ..................... 86

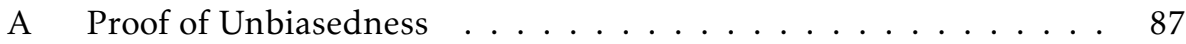

B Taylor and Unscented Transforms . . . . . . . . . . . . . . 88

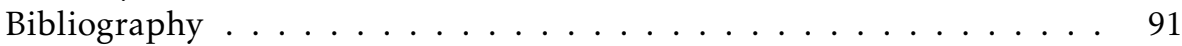

B Capacity estimation of two-dimensional channels using Sequential $\begin{array}{ll}\text { Monte Carlo } & 97\end{array}$

1 Introduction . . . . . . . . . . . . . . . . . 99

2 Two-dimensional channel models . . . . . . . . . . . . . . 100

2.1 Constrained channels and PGM . . . . . . . . . . . 101

2.2 High-dimensional undirected chains . . . . . . . . . 102

3 Sequential Monte Carlo . . . . . . . . . . . . . . . 102

3.1 Estimating the partition function using fully adapted SMC 103

3.2 SMC samplers and Forward Filtering/Backward Sampling 106

4 Experiments ..................... 107

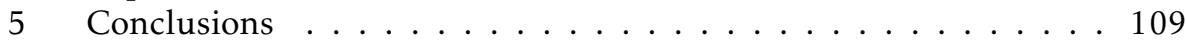

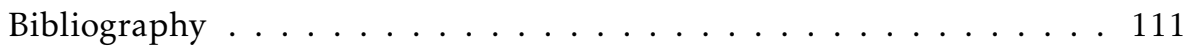

C Sequential Monte Carlo for Graphical Models $\quad 113$

1 Introduction . . . . . . . . . . . . . . . 115

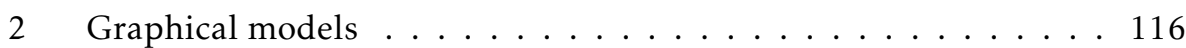

3 Sequential Monte Carlo . . . . . . . . . . . . . . 117

3.1 Sequential decomposition of graphical models . . . . . . . 117

3.2 Sequential Monte Carlo for PGMs . . . . . . . . . . . . . 119

3.3 Estimating the partition function . . . . . . . . . 120

4 Particle MCMC and partial blocking . . . . . . . . . . 121

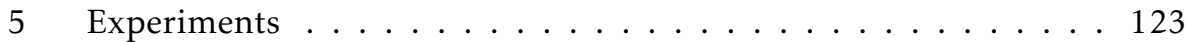

$5.1 \quad$ Classical XY model . . . . . . . . . . . . . . 123

5.2 Likelihood estimation in topic models . . . . . . . . . . 124

5.3 Gaussian MRF . . . . . . . . . . . . . 126

6 Conclusion ...................... 127 
Bibliography .......................... 128

D Nested Sequential Monte Carlo Methods $\quad 131$

1 Introduction . . . . . . . . . . . . . . . . 133

2 Background and Inference Strategy . . . . . . . . . . . . . 135

2.1 Sequential Monte Carlo . . . . . . . . . . . . . . . 135

2.2 Adapting the Proposal Distribution . . . . . . . . . . . 136

3 Proper Weighting and Nested Importance Sampling . . . . . . . 136

3.1 Exact Approximation of the Proposal Distribution . . . . . 137

3.2 Modularity of Nested IS . . . . . . . . . . . . . . . . 138

4 Nested Sequential Monte Carlo . . . . . . . . . . . . . . . . . . . . . . . . . . . . . . . .

4.1 Fully Adapted SMC Samplers . . . . . . . . . . . . . . . 139

$4.2 \quad$ Fully Adapted Nested SMC Samplers . . . . . . . . . . . . . 140

4.3 Backward Simulation and Modularity of NSMC . . . . . . . 141

5 Practicalities and Related Work . . . . . . . . . . . . . . 142

6 Experimental Results . . . . . . . . . . . . . . . . . . . . 144

6.1 Gaussian State Space Model . . . . . . . . . . . . . . . 144

6.2 Non-Gaussian State Space Model . . . . . . . . . . . . . . 145

6.3 Spatio-Temporal Model - Drought Detection . . . . . . . 146

Bibliography ...................... 149

E High-dimensional Filtering using Nested Sequential Monte Carlo 153

1 Introduction . . . . . . . . . . . . . . . 156

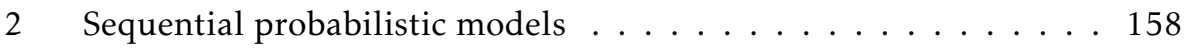

2.1 Markov random fields . . . . . . . . . . . . . 158

2.2 Spatio-temporal state space models . . . . . . . . . 160

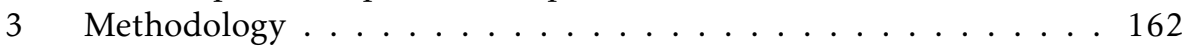

3.1 Fully Adapted Sequential Monte Carlo . . . . . . . . . . . 162

3.2 Leveraging Forward Filtering-Backward Simulation . . . . 163

3.3 Nested Sequential Monte Carlo . . . . . . . . . . . . . . . 164

3.4 Constructing $\eta_{t-1}^{M}, \tau_{t}$ and $\kappa_{t}^{M} \ldots \ldots \ldots 167$

3.5 Theoretical Justification . . . . . . . . . . . . . . . 169

3.6 Modularity and implementation aspects . . . . . . . 172

4 Numerical Results . . . . . . . . . . . . . . . . . . . . 173

4.1 Gaussian Model . . . . . . . . . . . . . . . 173

4.2 Soil Carbon Cycles . . . . . . . . . . . . . . . 173

4.3 Mixture Model . . . . . . . . . . . . . . 175

A General Nested Sequential Monte Carlo . . . . . . . . . . . . 176

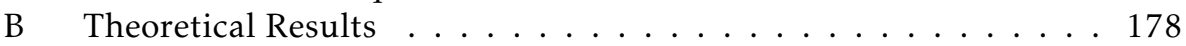

B.1 Proof of Theorem 1 ................. 178

B.2 Proof of Proposition $2 \ldots \ldots \ldots 182$

B.3 Proposition $3 \ldots \ldots \ldots 18 \ldots \ldots$

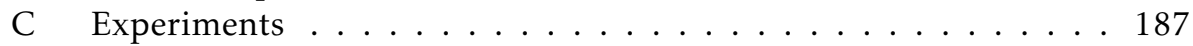

C.1 Comparison with Independent Resampling Particle Filter . 187

Bibliography ...................... 189 
F Interacting Particle Markov Chain Monte Carlo 195

1 Introduction . . . . . . . . . . . . . . . . . . . 197

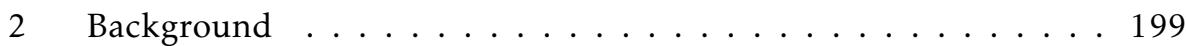

2.1 Sequential Monte Carlo . . . . . . . . . . . . . 199

2.2 Particle Gibbs . . . . . . . . . . . . . . 200

3 Interacting Particle Markov Chain Monte Carlo . . . . . . . . . 200

3.1 Theoretical Justification . . . . . . . . . . . . 203

3.2 Using All Particles . . . . . . . . . . . . . . . 204

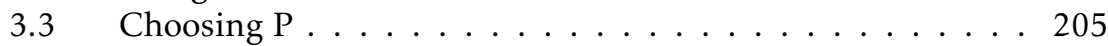

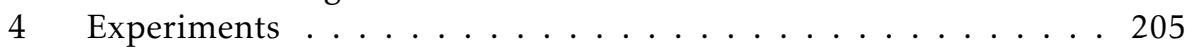

$4.1 \quad$ Linear Gaussian State Space Model . . . . . . . . . . . . 207

4.2 Nonlinear State Space Model . . . . . . . . . . . . . 208

5 Discussion and Future Work . . . . . . . . . . . . . . . 210

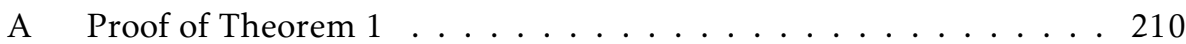

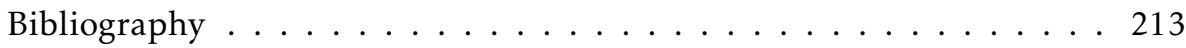

G Reparameterization Gradients through Acceptance-Rejection Sampling $\begin{array}{ll}\text { Algorithms } & 215\end{array}$

1 Introduction .................... 217

2 Variational Inference . . . . . . . . . . . . . . . . 219

3 Reparameterizing the Acceptance-Rejection Sampler . . . . . . . 220

3.1 Reparameterized Rejection Sampling . . . . . . . . . . . . . 221

3.2 The Reparameterized Rejection Sampler in Variational Inference .................... 222

$3.3 \quad$ Full Algorithm . . . . . . . . . . . . . . . . . 224

4 Related Work . . . . . . . . . . . . . . . . . 225

5 Examples of Acceptance-Rejection Reparameterization . . . . . . 226

5.1 Gamma Distribution . . . . . . . . . . . 226

5.2 Dirichlet Distribution . . . . . . . . . . . . 228

6 Experiments ....................... 229

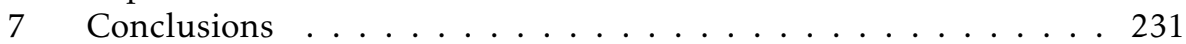

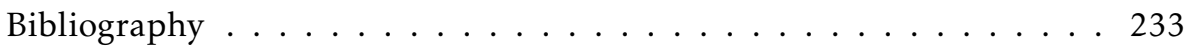

H Variational Sequential Monte Carlo 237

1 Introduction . . . . . . . . . . . . . . . . . 239

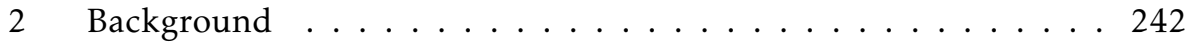

3 Variational Sequential Monte Carlo . . . . . . . . . . . . . . . 244

4 Perspectives on Variational SMC . . . . . . . . . . . . . . 248

5 Empirical Study . . . . . . . . . . . . . . . . . 250

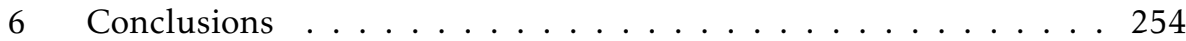

A Variational Sequential Monte Carlo - Supplementary Material . . 255

A.1 Proof of Proposition 1 . . . . . . . . . . . . 255

A.2 Proof of Theorem $1 \ldots \ldots \ldots 256$

A.3 Stochastic Optimization .............. 256

A.4 Scaling With Dimension . . . . . . . . . . . . . . 257

Bibliography ..................... 259 
Part I

\section{Background}





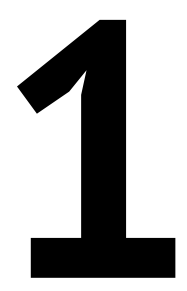

Introduction

Data and machine learning are by now an integral part of our everyday lives. Everytime we conduct a web search, machine learning ensures personalized and progressively better search results. Watching movies or listening to music? Machine learning is there to provide you with automatic recommendations on what to look at or listen to next. Need that sentence translated from English to Swedish? No problem! Use deep learning-powered machine translation. Machine learning is having an impact on everything from healthcare to video games. Wherever we have access to complex data, there is the potential that machine learning can be useful.

We are creating data at an unprecedented speed, quintillions of bytes every day. This is way more than any person could ever process in a lifetime. Machine learning instead uses computers and computer programs to help us make sense of the data. The increasing scale and complexity requires ever more efficient methods to process the data effectively.

With this motivation, the research in this thesis focuses on increasing the machine learning expert's toolbox and understanding of the tools available. We focus on stuyding the class of sequential Monte Carlo (SMC) methods for use in machine learning. We introduce the method in a tutorial article, study new applications within e.g. information theory and graphical models, develop methodological advances to SMC, and connect it to variational methods. 


\subsection{Data and machine learning}

We begin by giving some general background to what we mean when we talk about data and (machine) learning.

\subsubsection{Data}

Data is arguably the most important ingredient in machine learning. We need to study data to learn patterns and make predictions. Basically anything that can be stored or recorded can be considered to be data. Whilst data can have near arbitrary form, in this thesis we ultimately only consider data that is wellrepresented by numbers. To be concrete we provide below a few of the examples that appear in some form or other in Part II:

Images: By using intensity for grayscale or RGB values for color, we can represent images using numbers. One example that we study is how machine learning can be used to generate images. (see Paper G)

Text: We can represent text using e.g. integer values, corresponding to a word's location in a dictionary. We study, amongst other things, how we can automatically categorize and sort documents from just their textual content. (see Papers C and G)

Brain activity: One common way to represent data from a biological neural network is to use spikes of brain activity, e.g. the number of neurons firing as a function of time. We study dynamical models for motor cortex neurons in a macaque monkey. (see Paper $\mathrm{H}$ )

Forex: The exchange rate between two currencies is easily represented by a numerical value. We study the volatility, or degree of variation, of the exchange rate between the US dollar and various other currencies. (see $\mathrm{Pa}$ per $\mathrm{H}$ )

Precipitation: Average amount of precipitation in a given location during a given time period can also be represented using numerical values. We use observations of monthly average precipitation values collected over decades to detect extended periods of drought in North America and sub-Saharan Africa. (see Paper D)

To illustrate new methods for machine learning we also make liberal use throughout this thesis of artificially generated (simulated) data. Sometimes the data may mimick properties of real data, such as the soil carbon data from Paper E. Other times, the simulated data is purely artificial and only used to illustrate and profile the properties of the proposed algorithm, such as in Paper B. 


\subsubsection{Machine learning}

Learning can be thought of as distilling information, or improving performance at a task, based on data. Machine learning simply means there is a machine (or computer program) that, with more or less automation, does the actual learning.

One of the most succesful coherent approaches to machine learning in the presence of uncertainty is probabilistic machine learning (Ghahramani, 2015). Random variables and probabilities are used to relate the data to a mathematical model. The model includes latent variables, variables that we do not observe directly but nevertheless are interested in knowing the values of. We make use of inference to deduce these values based on the model and data. Based on the results of inference, we can take rational decisions or actions. We illustrate with a simple example where our data consists of recorded results of a sequence of coin flips (heads/tails):

Model: The model is a probability distribution describing the relation between data and (unobserved) latent variables.

Example: The latent variable is the probability of a flip resulting in heads.

Inference: We deduce the value, or range of potential values, for the latent variables given our observed data.

Example: What is our best guess for the value, or range of likely values, of the probability of heads?

Decision: Based on the inference result and available choices, we take a rational decision on the best action.

Example: Is the coin fair enough, probability of heads is close to one half, or should we use another coin?

Inference typically leads to mathematical equations that we can not write down a closed-form solution to. This means that we have use for methods that solve the inference task approximately, so called approximate inference methods.

We return to these subjects in Chapter 2 and Chapter 3, where we expand and formalize the concepts briefly introduced above.

\subsection{Contributions}

The main contribution of this thesis is developing new methods for approximate inference and learning in latent variable models.

This section will elaborate on the contributions, organized by different research themes. Each theme is introduces and summarized in its own subsection. Fur- 
thermore, the relevant papers to each theme is presented, as well as the author of this thesis' contributions to each particular paper.

\subsubsection{Elements of sequential Monte Carlo}

Sequential Monte Carlo methods are a powerful tool for approximate inference. The first contribution in Part II of this thesis is a self-contained tutorial article on SMC methods from a machine learning perspective.

The tutorial has a distinct focus from previously published tutorials on the topic. It focuses on the SMC algorithm's ability to approximate the final smoothing or marginal distribution of the latent variable model. These types of methods have recently seen use within machine learning to everything from improving variational inference to reinforcement learning.

This tutorial complements Part I, the background for this thesis.

\section{Relevant publications}

Paper A:

Christian A. Naesseth, Fredrik Lindsten, and Thomas B. Schön. Elements of sequential Monte Carlo. Foundations and Trends in Machine Learning, 2018b. (proposal accepted, manuscript in preparation).

The idea originated from the author of this thesis, and was subsequently refined in discussion with the co-authors. The majority of the article is written by the author of this thesis. All examples and code supplied have been implemented and written by the author of this thesis.

\subsubsection{Sequential Monte Carlo for graphical models}

A probabilistic graphical model (PGM) is a type of model where the conditional independencies of the joint probability distribution of the variables are described by edges in a graph. The graph structure allows for easier and stronger control on the type of prior information that the user can express. The main limitation of the PGM is that exact inference is typically intractable and approximate inference is difficult.

The relevant publications in Part II explores new applications for SMC inference to compute the capacity of two-dimensional information channels. The capacity of the channel can be expressed as the normalization constant of an undirected PGM. An example where this is potentially useful is in emerging technology such as optical data storage. Furthermore, a new way of applying SMC methods specifically suitable for generic PGMs is developed and studied. Potential applications 
include sampling from undirected PGMs, a notoriously difficult problem, generating efficient unbiased estimates of normalization constants, and evaluating model fit.

\section{Relevant publications}

Paper B:

Christian A. Naesseth, Fredrik Lindsten, and Thomas B. Schön. Capacity estimation of two-dimensional channels using sequential Monte Carlo. In IEEE Information Theory Workshop (ITW), pages 431-435, 2014a.

The idea originated from the author of this thesis, and was subsequently refined in discussion with the co-authors. The majority of the article is written by the author of this thesis. All empirical studies have been carried out by the author of this thesis.

\section{Paper C:}

Christian A. Naesseth, Fredrik Lindsten, and Thomas B. Schön. Sequential Monte Carlo for graphical models. In Advances in Neural Information Processing Systems (NIPS), pages 1862-1870, $2014 \mathrm{~b}$.

The idea originated from Fredrik Lindsten and Thomas B. Schön. The majority of the article is written by the author of this thesis. All empirical results, apart from the algorithm implementation for the latent Dirichlet allocation example, are by the author of this thesis.

Fredrik Lindsten, Adam M. Johansen, Christian A. Naesseth, Brent Kirkpatrick, Thomas B. Schön, John Aston, and Alexandre BouchardCôté. Divide-and-conquer with sequential Monte Carlo. Journal of Computational and Graphical Statistics, 26(2):445-458, 2017.

The author of this thesis has made minor contributions to the empirical results and writing of this paper. Because the author of this thesis has only made minor contributions to this paper it is not included in Part II.

\subsubsection{Nested sequential Monte Carlo}

The key design choice in any SMC algorithm is without a doubt the proposal distribution. The locally optimal proposal distribution is the best local proposal distribution that uses data only in a causal fashion, i.e. only up until the current iteration. Unfortunately this distribution is typically unavailable and approximations must be made for a practical SMC algorithm.

The first two relevant papers within this theme in Part II introduce so called exact approximations to any proposal distribution, focusing on the locally optimal proposal. The key idea is to construct a nested Monte Carlo (MC) method that approximates the proposal distribution, and then choose weights and samples in 
such a way such that the resulting estimate is still consistent. There is a significant methodological overlap in these two papers. However, they complement each other with distinct derivations, focus, and results.

The last relevant paper uses similar ideas as in nested MC methods, but now instead tackle the problem of parallelization of inference for generic probabilistic programming. A new algorithm, interacting particle Markov chain Monte Carlo, particularly suited for taking advantage of multi-core architecture is developed and studied.

\section{Relevant publications}

Paper D:

Christian Naesseth, Fredrik Lindsten, and Thomas Schön. Nested sequential Monte Carlo methods. In International Conference on Machine Learning (ICML), pages 1292-1301, 2015a.

The idea originated from discussions between the co-authors of the paper. The majority of the article is written by the author of this thesis. The proof of Theorem 2 is by Fredrik Lindsten. All empirical results are by the author of this thesis.

Paper E:

Christian A. Naesseth, Fredrik Lindsten, and Thomas B. Schön. High-

dimensional filtering using nested sequential Monte Carlo. arXiv:1612.09162, 2016.

The idea originated from discussions between the co-authors of the paper. The majority of the article is written by the author of this thesis. All theoretical and empirical results are by the author of this thesis.

Paper F:

Tom Rainforth, Christian A. Naesseth, Fredrik Lindsten, Brooks Paige, Jan-Willem Vandemeent, Arnaud Doucet, and Frank Wood. Interacting particle Markov chain Monte Carlo. In International Conference on Machine Learning (ICML), pages 2616-2625, 2016.

The idea originated from discussions between the author of this thesis and Fredrik Lindsten, subsequently refined by the co-authors of the paper. The majority of the method development is written by the author of this thesis. All theoretical results are by the author of this thesis. The empirical results of Figure 1 are by the author of this thesis.

\subsubsection{Variational Monte Carlo}

Variational methods are another powerful tool for approximate inference, turning the integration problem in inference into an optimization problem which we can solve more efficiently. Classical approaches have relied on so-called mean 
field approximations to the posterior to derive tractable coordinate ascent algorithms for the optimization procedure. To allow for more flexible and accurate posterior inferences one needs to resort to stochastic optimization.

The first paper and contribution within this theme develops low-variance reparameterization gradients for a class of variational approximations that rely on the rejection sampler for simulation. The second paper combines variational methods with SMC, viewing the (expected) posterior distribution approximation from SMC as the variational approximation we need to optimize. This leads to a more flexible and accurate distribution, trading off fidelity to the posterior with computational cost.

\section{Relevant publications}

\section{Paper G:}

Christian A. Naesseth, Francisco Ruiz, Scott W. Linderman, and David M. Blei. Reparameterization gradients through acceptance-rejection sampling algorithms. In Artificial Intelligence and Statistics (AISTATS), pages 489-498, 2017.

The idea originated from discussions between the co-authors of the paper. The majority of the article is written by the author of this thesis. All theoretical and empirical results are by the author of this thesis.

\section{Paper H:}

Christian A. Naesseth, Scott W. Linderman, Rajesh Ranganath, and David M. Blei. Variational sequential Monte Carlo. In Artificial Intelligence and Statistics (AISTATS), pages 968-977, 2018a.

The idea originated from the author of this thesis, and was subsequently refined in discussion with the co-authors. The majority of the article is written by the author of this thesis. All theoretical and empirical results are by the author of this thesis.

\subsection{Thesis outline}

The thesis is divided into two parts. The first part contains a brief introduction to machine learning from a probabilistic or statistical point of view, explaining the three central concepts modeling, inference, and decision making. The first part concludes with an introduction to the two main methods for performing approximate inference, variational and Monte Carlo methods. The second part contains edited versions of eight publications. 


\subsection{Publications}

The author's published work is listed below in reverse chronological order. Publications indicated by $\mathrm{a} \star$ are included in Part II of this thesis.

$\star$ Christian A. Naesseth, Fredrik Lindsten, and Thomas B. Schön. Elements of sequential Monte Carlo. Foundations and Trends in Machine Learning, 2018b. (proposal accepted, manuscript in preparation).

$\star$ Christian A. Naesseth, Scott W. Linderman, Rajesh Ranganath, and David M. Blei. Variational sequential Monte Carlo. In Artificial Intelligence and Statistics (AISTATS), pages 968-977, 2018a.

Christian A. Naesseth, Francisco Ruiz, Scott W. Linderman, and David M. Blei. Reparameterization gradients through acceptance-rejection sampling algorithms. In Artificial Intelligence and Statistics (AISTATS), pages 489-498, 2017.

Fredrik Lindsten, Adam M. Johansen, Christian A. Naesseth, Brent Kirkpatrick, Thomas B. Schön, John Aston, and Alexandre BouchardCôté. Divide-and-conquer with sequential Monte Carlo. Journal of Computational and Graphical Statistics, 26(2):445-458, 2017.

Sina Khoshfetrat Pakazad, Christian A. Naesseth, Fredrik Lindsten, and Anders Hansson. Distributed, scalable and gossip-free consensus optimization with application to data analysis. arXiv:1705.02469, 2017.

$\star$ Christian A. Naesseth, Fredrik Lindsten, and Thomas B. Schön. Highdimensional filtering using nested sequential Monte Carlo. arXiv:1612.09162, 2016.

^ Tom Rainforth, Christian A. Naesseth, Fredrik Lindsten, Brooks Paige, Jan-Willem Vandemeent, Arnaud Doucet, and Frank Wood. Interacting particle Markov chain Monte Carlo. In International Conference on Machine Learning (ICML), pages 2616-2625, 2016.

Christian A. Naesseth, Fredrik Lindsten, and Thomas B. Schön. Towards automated sequential Monte Carlo for probabilistic graphical models. In NIPS Workshop on Black Box Learning and Inference, 2015b.

Thomas B. Schön, Fredrik Lindsten, Johan Dahlin, Johan Wågberg, Christian A. Naesseth, Andreas Svensson, and Liang Dai. Sequential Monte Carlo methods for system identification. IFAC-PapersOnLine (SYSID), 48(28):775-786, 2015.

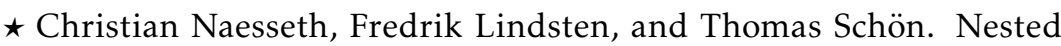
sequential Monte Carlo methods. In International Conference on Machine Learning (ICML), pages 1292-1301, 2015a. 
$\star$ Christian A. Naesseth, Fredrik Lindsten, and Thomas B. Schön. Sequential Monte Carlo for graphical models. In Advances in Neural Information Processing Systems (NIPS), pages 1862-1870, $2014 \mathrm{~b}$.

$\star$ Christian A. Naesseth, Fredrik Lindsten, and Thomas B. Schön. Capacity estimation of two-dimensional channels using sequential Monte Carlo. In IEEE Information Theory Workshop (ITW), pages 431-435, $2014 a$. 



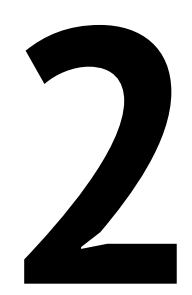

\section{Probabilistic machine learning}

Machine learning is a science that focuses on the study and design of computer programs that learn automatically from data and experience. Probabilistic (or statistical) machine learning does this while explicitly representing uncertainty. With probabilistic machine learning we make use of probability theory to represent the uncertainty. Uncertainty, for the purpose of this thesis, can take many forms. It can be an inherent randomness in a system, or it could be about our own imperfect or partial information about the system we observe. This is also tightly connected to the definition of what probabilities and random variables actually are. We take a very pragmatic approach, making use of ideas from both Bayesian and frequentist perspectives on probability.

In this chapter we introduce some fundamentals to probabilistic machine learning: the concepts of modeling, inference, and decision. Modeling is the procedure of writing down explicit assumptions on how the data was generated, usually in the form of a probability distribution. Inference means taking our model and combining it with our observed data to reach a conclusion; perhaps to predict future data or answer a causal question. Decision is using our inferences to make a choice.

The field of machine learning is closely related to, amongst other fields, computational statistics (Efron and Hastie, 2016) and mathematical optimization (Bertsekas, 2016; Nocedal and Wright, 2006). Throughout this thesis, we will see how making use of methods from these fields will empower us with more powerful methods for machine learning.

This chapter is intentionally kept quite brief. For a more thorough introduction to probabilistic and statistical machine learning, we refer the reader to the textbooks by Bishop (2006) or Friedman et al. (2009). 


\subsection{Modeling}

A model describes data from a system that we observe. We will focus on the use of probabilistic models: mathematical models making use of probability distributions to encode uncertainty. The key variables of the model are the data $y$ and the parameters $\theta$, a type of latent (unobserved) variable. The data y usually consists of $T$ separate observations $\mathbf{y}=\left\{y_{t}\right\}_{t=1}^{T}$ indexed by $t$.

To prior, or not to prior One fundamental consideration is whether we define a joint probability of both data and parameters, i.e. $p(\mathbf{y}, \theta)=p(\mathbf{y} \mid \theta) p(\theta)$, or if we focus solely on the likelihood $p(\mathbf{y} \mid \theta)$. Pragmatically the two differ in the prior $p(\theta)$ and the interpretation of the parameters. For frequentist statistics we are interested in an estimator of $\theta$ that works well for repeated use, where we could potentially observe many different realisations of $\mathbf{y}$. In Bayesian statistics, we assign a prior degree of belief for the potential values of the parameter $\theta$ and interpret the parameter as a random variable. Then based on the observed data, a particular realization of $\mathbf{y}$, we update our belief about the parameter $\theta$. Bayesian statistics is usually criticized for being subjective because of the prior, which introduces preferences for different values of the parameters. These priors can be different for different people, meaning that the inference can also be different. However, the likelihood (common to both approaches) is also a subjective choice. Either way, we will see both of these types of interpretations on unknown parameters in Part II, with a focus on the Bayesian approach.

For a more thorough discussion of the fundamentals of the different schools of statistical inference we recommend Casella and Berger (2002); Gelman et al. (2013); Robert (2007).

Example We present a simple toy example in Example 2.1, the data is modeled as independent and identically distributed from a normal distribution with unknown mean. This example will be used throughout this chapter to illustrate the different concepts.

\section{Example 2.1: Toy Example: Model}

For illustrative purposes we will consider a very simple model for which inference and decision is usually analytically tractable. We simulate data from the following joint probability

$$
p(\mathbf{y}, \theta)=p(\mathbf{y} \mid \theta) p(\theta)=\mathcal{N}(\theta \mid 0,1) \cdot \prod_{t=1}^{3} \mathcal{N}\left(y_{t} \mid \theta, 1\right),
$$

where $\mathcal{N}\left(\cdot \mid \mu, \sigma^{2}\right)$ denotes a normal distribution with mean $\mu$ and variance $\sigma^{2}$. We will use the dataset $y_{1}=-0.65, y_{2}=0.072$, and $y_{3}=-0.54$. 
Local latent variables A common technique to make modeling and inference easier is introducing extra (local) latent variables, also known as data augmentation (Van Dyk and Meng, 2001). This means that we have extra latent variables $\mathbf{x}=\left\{x_{t}\right\}_{t=1}^{T}$, where each local latent variable $x_{t}$ is often associated with a data point $y_{t}$. With this we can define the likelihood

$$
p(\mathbf{y} \mid \theta)=\int p(\mathbf{y}, \mathbf{x} \mid \theta) \mathrm{d} \mathbf{x}
$$

where $p(\mathbf{y}, \mathbf{x} \mid \theta)$ is known as the complete data likelihood. We present three examples of model classes that are ubiquitous in practice:

- Conditionally independent model: In the conditionally independent model the complete data likelihood will satisfy the following factorization

$$
p(\mathbf{y}, \mathbf{x} \mid \theta)=\prod_{t=1}^{T} p\left(x_{t} \mid \theta\right) p\left(y_{t} \mid x_{t}, \theta\right),
$$

i.e. the data are conditionally independent given $\theta$. This is a common model for exchangeable data (Gelman et al., 2013): data $y_{t}, t=1, \ldots, T$ where a reordering does not change the joint distribution $p(\mathbf{y})$. Examples of this type are used in Papers G and H.

- State space model: The local latent variables in a state space model (SSM) satisfy a Markov property (Cappé et al., 2005). This means that the prior for $x_{t}$ only depends on its immediate preceding latent variable $x_{t-1}$, and the data $y_{t}$ only directly depends on $x_{t}$. With these assumptions we get

$$
p(\mathbf{y}, \mathbf{x} \mid \theta)=p\left(x_{1} \mid \theta\right) p\left(y_{1} \mid x_{1}, \theta\right) \prod_{t=2}^{T} p\left(x_{t} \mid x_{t-1}, \theta\right) p\left(y_{t} \mid x_{t}, \theta\right) .
$$

Examples of this type are used in Papers $\mathrm{E}$ and $\mathrm{H}$.

- Probabilistic graphical model: The local latent variables in a PGM (Koller et al., 2009) have a dependence defined by a graph. The complete data likelihood for the model, based on a graph with edges defined in the edge set $\mathcal{E}$, can be written as

$$
p(\mathbf{y}, \mathbf{x} \mid \theta)=\frac{1}{Z(\theta)} \prod_{(i, j) \in \mathcal{E}} \psi\left(x_{i}, x_{j} \mid \theta\right) \prod_{t=1}^{T} \phi\left(x_{t}, y_{t} \mid \theta\right),
$$

where $Z(\theta)$ is the normalization constant, ensuring that the right-hand side is a probability distribution. The positive functions $\psi, \phi$ are known as interaction and observation potentials, respectively. Examples of this type are used in Papers B, C, D, and E.

Local latent-variable-based models are mainstay modeling tool used throughout the field of probabilistic machine learning. 
Mechanistic-algorithmic continuum One way to distinguish different models on a high level is on a mechanistic-algorithmic continuum. On the one side, purely mechanistic models are fully specified models based on physical or natural processes where the parameters have a physical or natural interpretation. An example of this type of model is Newton's second law of motion. On the other side, we have the purely algorithmic model where the parameters have no physical or natural interpretation and we are only interested in its predictive abilities. An example that comes close to this are models making use of (artificial) neural networks and deep learning. Machine learning methods tend to make use of models that fall closer to the algorithmic part of the spectrum. However, no matter where a specific model falls within this continuum, we want to infer its parameters and other unknown latent variables.

\subsection{Inference}

Inference means taking the observed data and combining it with our model assumptions to deduce properties on the latent variables. The goal of inference usually takes one of two forms: we are interested in either prediction or causality. Prediction means learning to forecast future values of new, as of yet unobserved, data. Causality focuses on understanding the parameter, the values it takes, and how it affects the data. Our main focus in this thesis is predictive inference based on the posterior distribution and the maximum likelihood estimator.

Posterior distribution When we have a joint probabilistic model for both data and parameters, inference is conceptually straightforward. By Bayes' rule, a fundamental result of conditional probabilities, the posterior distribution of $\theta$ given $y$ is

$$
p(\theta \mid \mathbf{y})=\frac{p(\mathbf{y} \mid \theta) p(\theta)}{p(\mathbf{y})}
$$

where $p(\mathbf{y})=\int p(\mathbf{y}, \theta) \mathrm{d} \theta$ is known as the marginal likelihood. The posterior distribution is the fundamental object for Bayesian inference. It is then used both in prediction and causality to compute expectations of functions $f(\theta)$ with respect to the posterior,

$$
\mathbb{E}_{p(\theta \mid \mathbf{y})}[f(\theta)]=\int f(\theta) p(\theta \mid \mathbf{y}) \mathrm{d} \theta .
$$

For example, when we want to predict new potential values $y^{\star}$ for our data we can use the posterior predictive distribution. This distribution can be written as an expectation of $\mathrm{f}(\theta)=p\left(y^{\star} \mid \theta\right)$ with respect to the posterior distribution,

$$
p\left(y^{\star} \mid \mathbf{y}\right)=\int p\left(y^{\star} \mid \theta\right) p(\theta \mid \mathbf{y}) \mathrm{d} \theta
$$


Bayesian statistics and the posterior distribution can be traced back to early work by the English statistician and reverend Thomas Bayes (1701-1761) and the French mathematician Pierre-Simon Laplace (1749-1827) (Bayes, 1763; Laplace, 1774; Stigler, 1986). Our current interpretation of Bayesian probability has its root in Laplace's extensive work on subjective probability.

Maximum likelihood When we only have access to the likelihood function, we can take several approaches to find a good value of the parameter. One of the most common and sensible approaches is to maximize the likelihood $p(\mathbf{y} \mid \theta)$ with respect to the parameters: the maximum likelihood parameter estimate $\widehat{\theta}^{\mathrm{ML}}$ is

$$
\widehat{\theta}^{\mathrm{ML}}=\underset{\theta}{\arg \max } \log p(\mathbf{y} \mid \theta) .
$$

The logarithm is introduced purely for computational convenience. Since it is a monotone function of its argument, it does not change the maximizing argument $\widehat{\theta}^{\mathrm{ML}}$.

Prediction in the maximum likelihood framework focuses on using the likelihood evaluated at the maximum likelihood value for the parameters, i.e. $p\left(y^{\star} \mid \widehat{\theta}^{\mathrm{ML}}\right)$.

The rise and popularization of frequentist statistics and maximum likelihood estimators can be traced back mainly to work by the British statistician Sir Ronald Fisher (1890-1962) during the early 20th century (Fisher, 1922). However, the principle and idea had previously been used by Hagen, Gauss, and Edgeworth (Hald, 1999).

Example We return to our Gaussian toy example to perform Bayesian and frequentist inference, see Example 2.2. We focus on the posterior distribution and maximimum likelihood estimate.

Example 2.2: Toy Example: Inference

Under the model assumptions of Example 2.1 we can analytically solve for the posterior distribution and maximum likelihood parameter:

- Posterior distribution:

$$
p(\theta \mid \mathbf{y})=\mathcal{N}\left(\theta \mid \frac{1}{T+1} \sum_{t=1}^{T} y_{t}, \frac{1}{T+1}\right)
$$

- Maximum likelihood:

$$
\widehat{\theta}^{\mathrm{ML}}=\frac{1}{T} \sum_{t=1}^{T} y_{t}
$$




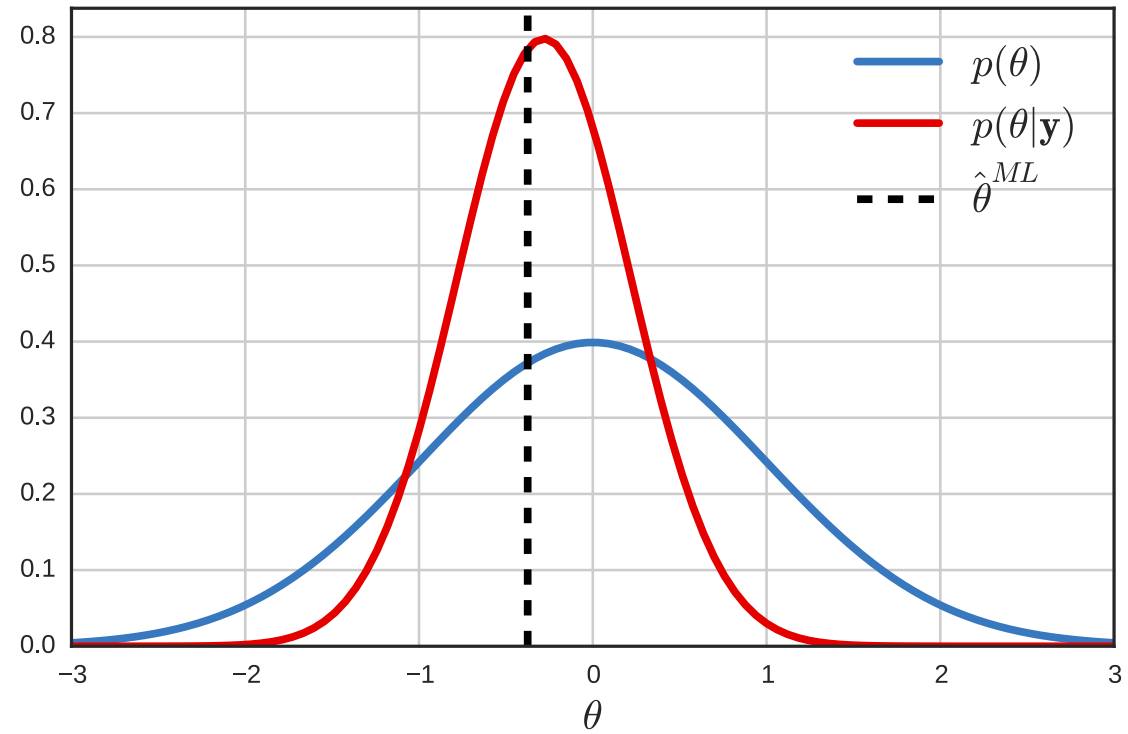

Figure 2.1: The prior distribution $p(\theta)$, posterior distribution $p(\theta \mid \mathbf{y})$, and maximum likelihood estimate $\widehat{\theta}^{M L}$, for the dataset $y_{1}=-0.65, y_{2}=0.072$, and $y_{3}=-0.54$ based on our Gaussian model.

With our example dataset $y_{1}=-0.65, y_{2}=0.072$, and $y_{3}=-0.54$ we illustrate the model, posterior distribution, and maximum likelihood estimator in Figure 2.1. The true value of the parameter that generated this particular dataset is $\theta^{\star}=$ -0.78 .

We have seen a simple example were exact inference is analytically tractable, i.e. closed-form solutions exist. However, this will not be the case in general. Most models and data that we encounter in practice will lead to an intractable inference problem, i.e. one where we can not evaluate the posterior distribution or find the maximum likelihood value. For the posterior distribution it is usually the marginal likelihood that is computationally intractable, because it requires us to evaluate a (potentially) high-dimensional integral, which is difficult. This means that we will have to resort to some form of approximation, which is the topic of Chapter 3 and the publications in Part II of this thesis.

\subsection{Decision}

Decision theory is associated with the notion of risk and loss. The typical setting is that we are interested in making a choice for the value of parameter $\theta$, by picking an estimator $\delta(\mathbf{y})$ that approximates the parameter of interest as well as possible. To do this we first define the concepts of loss and risk. 
The loss $\mathrm{L}(\theta, \delta)$ is a non-negative function of the parameter and decision. It lets us evaluate the penalty of taking the decision $\delta$ when the parameter is in fact $\theta$. The risk $\mathrm{R}(\cdot)$ defines the average loss that we would like to optimize with respect to the decision $\delta$. The risk differs between a frequentist and Bayesian interpretation, by changing what is interpreted as random and what is fixed.

Frequentist risk: In the frequentist approach to decision theory, we average the loss for the potential datasets we could have observed. The frequentist risk is defined by computing the expectation of our loss with respect to the likelihood, i.e.

$$
\mathrm{R}(\theta, \delta)=\int \mathrm{L}(\theta, \delta(\mathbf{y})) p(\mathbf{y} \mid \theta) \mathrm{d} \mathbf{y} .
$$

The frequentist risk is a function not only of our decision $\delta$, but also of our (unknown) parameter $\theta$. The actual observed data is not taken into account any further, instead we average over the likelihood and any potential dataset we could see if the experiment was repeated.

Bayes risk: With the Bayesian approach we instead integrate over our uncertainty on the parameters. The posterior expected loss is given by

$$
\mathrm{r}(\delta(\mathbf{y}), \mathbf{y})=\int \mathrm{L}(\theta, \delta(\mathbf{y})) p(\theta \mid \mathbf{y}) \mathrm{d} \theta,
$$

which is a function of the observed data $\mathbf{y}$ and our decision $\delta$. The Bayes estimator is given by

$$
\delta^{\mathrm{B}}(\mathbf{y}):=\underset{\delta}{\arg \min } \mathrm{r}(\delta(\mathbf{y}), \mathbf{y})
$$

for each dataset $\mathbf{y}$ that we could potentially observe. Unlike the frequentist risk, the posterior expected loss only depends on the model and dataset $\mathbf{y}$ (which are known).

The expected posterior loss in Equation (2.12) is the only risk we care about from a strictly Bayesian point of view. All calculations are conditional on our known data. However, with the prior we can connect the posterior expect loss to the frequentist risk by defining the integrated risk,

$$
\mathrm{R}(\delta)=\int \mathrm{r}(\delta(\mathbf{y}), \mathbf{y}) p(\mathbf{y}) \mathrm{d} \mathbf{y}=\int \mathrm{R}(\theta, \delta) p(\theta) \mathrm{d} \theta,
$$

where $p(\theta)$ is the prior distribution and $p(\mathbf{y})$ is the marginal likelihood. It is possible to show that $\delta^{\mathrm{B}}$ is the estimator that minimizes the integrated risk (Robert, 2007). The Bayes risk is then defined by $\mathrm{R}\left(\delta^{\mathrm{B}}\right)$. If the Bayes risk is finite we call $\delta^{\mathrm{B}}$ admissible. It dominates and outperforms any other estimator in terms of frequentist risk. 
For the reader interested in more information about the foundations of Bayesian decision theory, and its connection to the frequentist approach, we refer to Robert (2007).

Example Returning to the toy example we consider two loss functions: the squared error and absolute error losses. See Example 2.3 for a derivation of the optimal Bayesian decisions in this case.

Example 2.3: Toy Example: Decision

Using the model definition in Example 2.1 and the posterior distribution from Example 2.2, we can derive the optimal Bayes estimators for the two loss functions:

- Squared error: $\mathrm{L}(\theta, \delta)=(\theta-\delta(\mathbf{y}))^{2}$

For the squared error loss we obtain the Bayes estimator

$$
\delta^{\mathrm{B}}(\mathbf{y})=\int \theta p(\theta \mid \mathbf{y}) \mathrm{d} \theta
$$

i.e. the posterior mean. For the dataset in our toy example, we get

$$
\delta^{\mathrm{B}}(\mathbf{y})=\frac{1}{4} \sum_{t=1}^{3} y_{t}=-0.28 .
$$

- Absolute error: $\mathrm{L}(\theta, \delta)=|\theta-\delta(\mathbf{y})|$

For the absolute error loss, we obtain the posterior median as the Bayes estimator

$$
\delta^{\mathrm{B}}(\mathbf{y})=\hat{\delta}, \quad \text { where } \mathbb{P}(\theta \leq \hat{\delta} \mid \mathbf{y})=\mathbb{P}(\theta \geq \hat{\delta} \mid \mathbf{y})=\frac{1}{2}
$$

where $\mathbb{P}(\theta \leq \hat{\delta} \mid \mathbf{y})=\int_{-\infty}^{\hat{\delta}} p(\theta \mid \mathbf{y}) \mathrm{d} \theta$ is the probability that the parameter $\theta$ is smaller than $\hat{\delta}$ given the data $\mathbf{y}$. For the dataset in our toy example we get

$$
\delta^{\mathrm{B}}(\mathbf{y})=\frac{1}{4} \sum_{t=1}^{3} y_{t}=-0.28,
$$

since the median and mean of the normal distribution coincide.

Decision theory does not feature prominently in this thesis; we focus instead on the task of approximate inference. However, as we can see above, being able to evaluate the posterior distribution (and expectations with respect to it) is key for Bayesian decision theory. This means that even in this setting, we have a need for efficient and accurate approximations to the posterior distribution. This is the main focus of most publications in Part II. 


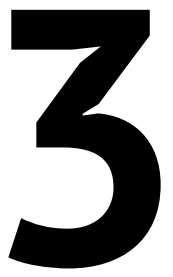

\section{Approximate inference}

Approximate inference is mainly focused on developing, and studying, approaches to estimate the posterior distribution. We can then make use of the approximation of the posterior and compute expectations with respect to it. We have seen from Chapter 2 that this is central to probabilistic machine learning. Approximate inference lets us trade off fidelity of the posterior approximation with computational complexity; the accuracy of the approximation typically depends on the amount of computation used.

Variational and Monte Carlo methods are two of the most popular approaches to approximate inference in machine learning. We will focus this chapter on introducing these two types of methods. First, we give a short introduction to Monte Carlo methods. These methods use (pseudo-)random numbers, usually referred to as samples, approximately distributed according to the posterior distribution. The samples are then averaged to estimate the posterior and posterior expectations. Second, we introduce and explain variational methods for approximate inference. Variational methods postulate a family of approximating distributions, e.g. the normal distribution family parameterized by the mean and variance. We then use a suitable cost function to optimize the parameters such that the variational approximation fits as close as possible to the true distribution.

This chapter is intentionally kept brief. For a more thorough introduction to Monte Carlo and variational methods, we refer the reader to Liu (2004); Robert and Casella (2004), Paper A, Blei et al. (2017), and Bishop (2006). 


\subsection{Monte Carlo methods}

Monte Carlo methods are a class of algorithms relying on (pseudo-)random numbers to approximate high-dimensional integrals. As discussed in Eckhardt (1987), the roots of Monte Carlo methods can be found in work by the Polish-American scientist Stanislaw Ulam (1909-1984) and Hungarian-American mathematician John von Neumann (1903-1957) during the 1940s.

We will here focus on the basic idea behind the various MC methods, and explain rejection sampling which is used in Paper G. The remaining papers in Part II rely on importance sampling (IS) and SMC methods, which we give a thorough introduction to in Paper A.

\subsubsection{The Monte Carlo idea}

We have shown that probabilistic machine learning relies on performing inference. In the Bayesian approach this means that we are interested in estimating the posterior distribution or compute expectations with respect to it, i.e.

$$
\mathbb{E}_{p(\theta \mid \mathbf{y})}[\mathrm{f}(\theta)]=\int \mathrm{f}(\theta) p(\theta \mid \mathbf{y}) \mathrm{d} \theta .
$$

The key Monte Carlo idea (see e.g. Metropolis and Ulam (1949) for an early reference discussing the idea) is to draw samples, random numbers, that are either exactly or approximately distributed according to $p(\theta \mid \mathbf{y})$ and estimate the expectation by averaging

$$
\mathbb{E}_{p(\theta \mid \mathbf{y})}[\mathrm{f}(\theta)] \approx \frac{1}{N} \sum_{i=1}^{N} \mathrm{f}\left(\theta^{i}\right), \quad \theta^{i} \sim p(\theta \mid \mathbf{y}), i=1, \ldots, N
$$

We can view the samples $\left\{\theta^{i}\right\}_{i=1}^{N}$ as defining an empirical distribution that approximates the posterior,

$$
p(\mathrm{~d} \theta \mid \mathbf{y}) \approx \frac{1}{N} \sum_{i=1}^{N} \delta_{\theta^{i}}(\mathrm{~d} \theta)
$$

where $\delta_{\theta^{i}}(\mathrm{~d} \theta)$ is the Dirac measure at $\theta=\theta^{i}$.

This basic Monte Carlo method has many favorable properties. It is

- unbiased:

$$
\mathbb{E}\left[\frac{1}{N} \sum_{i=1}^{N} \mathrm{f}\left(\theta^{i}\right)\right]=\mathbb{E}_{p(\theta \mid \mathbf{y})}[\mathrm{f}(\theta)]
$$


- consistent:

$$
\frac{1}{N} \sum_{i=1}^{N} \mathrm{f}\left(\theta^{i}\right) \stackrel{\text { a.s. }}{\longrightarrow} \mathbb{E}_{p(\theta \mid \mathbf{y})}[\mathrm{f}(\theta)], \quad N \rightarrow \infty
$$

- asymptotically normal:

$$
\frac{\sqrt{N}}{\sigma_{\mathrm{f}}}\left(\frac{1}{N} \sum_{i=1}^{N} \mathrm{f}\left(\theta^{i}\right)-\mathbb{E}_{p(\theta \mid \mathbf{y})}[\mathrm{f}(\theta)]\right) \stackrel{d}{\rightarrow} \mathcal{N}(0,1), \quad N \rightarrow \infty,
$$

where $\stackrel{\text { a.s. }}{\longrightarrow}$ and $\stackrel{d}{\longrightarrow}$ denotes convergence almost surely and convergence in distribution, respectively. The asymptotic normality holds if the variance of the function $\mathrm{f}(\cdot)$ with respect to the posterior distribution $p(\theta \mid \mathbf{y}), \sigma_{\mathrm{f}}^{2}=\operatorname{Var}_{p(\theta \mid \mathbf{y})}(\mathrm{f}(\theta))$, is finite. One of the fundamental strengths of MC methods is that the rate of improvement as a function of the number of samples $N$, illustrated by the central limit theorem (asymptotic normality), does not depend on the dimensionality of the parameter $\theta$.

In practice it is often difficult or impossible to simulate exactly from the posterior distribution. In the next section we describe rejection sampling, a method that accomplishes exact simulation using auxiliary variables and samples from a different distribution.

\subsubsection{Rejection sampling}

Rejection sampling (Devroye, 1986; von Neumann, 1951), or acceptance-rejection sampling, is a method for exact simulation. In most cases we use a rejection sampler to generate random samples from a distribution for which standard sampling methods, such as inverse transform sampling (Robert and Casella, 2004), are unavailable or impractical. For notational convenience we will simply denote any distribution we are interested in generating samples from as $\gamma(\theta)$. We will refer to this as the target distribution.

The fundamental idea in rejection sampling is based on the straightforward idea that we can rewrite any density as an integral

$$
\gamma(\theta)=\int_{0}^{\gamma(\theta)} 1 \mathrm{~d} u
$$

for some auxiliary variable $u$. This means that the distribution $\gamma(\theta)$ is the marginal density of the uniform distribution on $\{(\theta, u): 0<u<\gamma(\theta)\}$, which we denote by $\mathrm{U}(\{(\theta, u): 0<u<\gamma(\theta)\})$. This corresponds to putting a uniform distribution on the area under the graph defined by the $\theta$-axis and $\gamma(\theta)$, see the shaded area in Figure 3.1 for an example. 
Now, if we could sample $(\theta, u) \sim \mathrm{U}(\{(\theta, u): 0<u<\gamma(\theta)\})$ we would have essentially solved the problem. However, directly trying to sample this distribution can be difficult. The straightforward way would be $\theta \sim \gamma(\theta)$ and $u \sim \mathrm{U}(0, \gamma(\theta))$. Because this approach relies on sampling $\theta$ from $\gamma(\theta)$ it defeats the purpose. What we can do instead is to sample uniformly over a larger area that covers the one we are interested in. Then we simply keep only the ones that fall within the area of interest defined by the constraint $0<u<\gamma(\theta)$ and reject the rest.

To accomplish this we need to make use of a proposal distribution $q(\theta)$. We assume that the proposal is simple to sample from, that we can evaluate $q(\theta)$, and that we can find a finite constant $M \geq 1$ such that $\gamma(\theta) \leq M q(\theta)$ for all values $\theta$. We generate samples from the distribution

$$
\left(\theta^{\prime}, u^{\prime}\right) \sim \mathrm{U}(\{(\theta, u): 0<u<M q(\theta)\}),
$$

simply by first simulating $\theta^{\prime} \sim q(\theta)$, and then $u^{\prime} \sim \mathrm{U}\left(0, M q\left(\theta^{\prime}\right)\right)$. Then we accept the sample $\left(\theta^{\prime}, u^{\prime}\right)$ if $u^{\prime}<\gamma\left(\theta^{\prime}\right)$, and otherwise repeat the procedure. We summarize: repeat

$$
\theta^{\prime} \sim q(\theta), \quad u^{\prime} \mid \theta^{\prime} \sim \mathrm{U}\left(0, M q\left(\theta^{\prime}\right)\right)
$$

until $u^{\prime}<\gamma\left(\theta^{\prime}\right)$. The accepted value $\theta^{\prime}$ is then distributed $\theta^{\prime} \sim \gamma(\theta)$ as required. We only have to know the desired $\gamma(\theta)$ up to a normalization constant $Z$, i.e. $\gamma(\theta)=\frac{1}{Z} \tilde{\gamma}(\theta)$. The normalization constant $Z$ may be subsumed into the factor $M$. In Bayesian inference we would have $\tilde{\gamma}(\theta)=p(\mathbf{y}, \theta)$, and the normalization constant $Z=p(\mathbf{y})$ would be subsumed by our choice of $M$ such that $p(\mathbf{y}, \theta) \leq$ $\operatorname{Mq}(\theta)$.

We illustrate rejection sampling with a simple example.

\section{Example 3.1: Rejection sampling}

We consider a simple scalar example. The probability distribution $\gamma(\theta)$ is defined by

$$
\gamma(\theta)=\frac{1}{Z} \underbrace{e^{-\frac{1}{2} \theta^{2}}\left(1+\sin ^{2}(4 \theta)+3 \cos ^{2}(\theta) \sin ^{2}(\theta)\right)}_{\tilde{\gamma}(\theta)},
$$

and we use the standard normal, which we can easily generate samples from, as our proposal distribution

$$
q(\theta)=\mathcal{N}(\theta \mid 0,1)=\frac{1}{\sqrt{2 \pi}} e^{-\frac{1}{2} \theta^{2}} .
$$

If we choose $M=2$ we have that $\gamma(\theta) \leq M \cdot q(\theta)$ for all $\theta$. We illustrate the setup in Figure 3.1. We found this value of $M$ by numerically computing $Z$. If we only assume that we can evaluate the unnormalized distribution $\tilde{\gamma}(\theta)$, we could instead choose e.g. $M=5 \sqrt{2 \pi}$. Higher values for $M$ will give rise to a lower acceptance probability, requiring us to propose more samples (on average). 


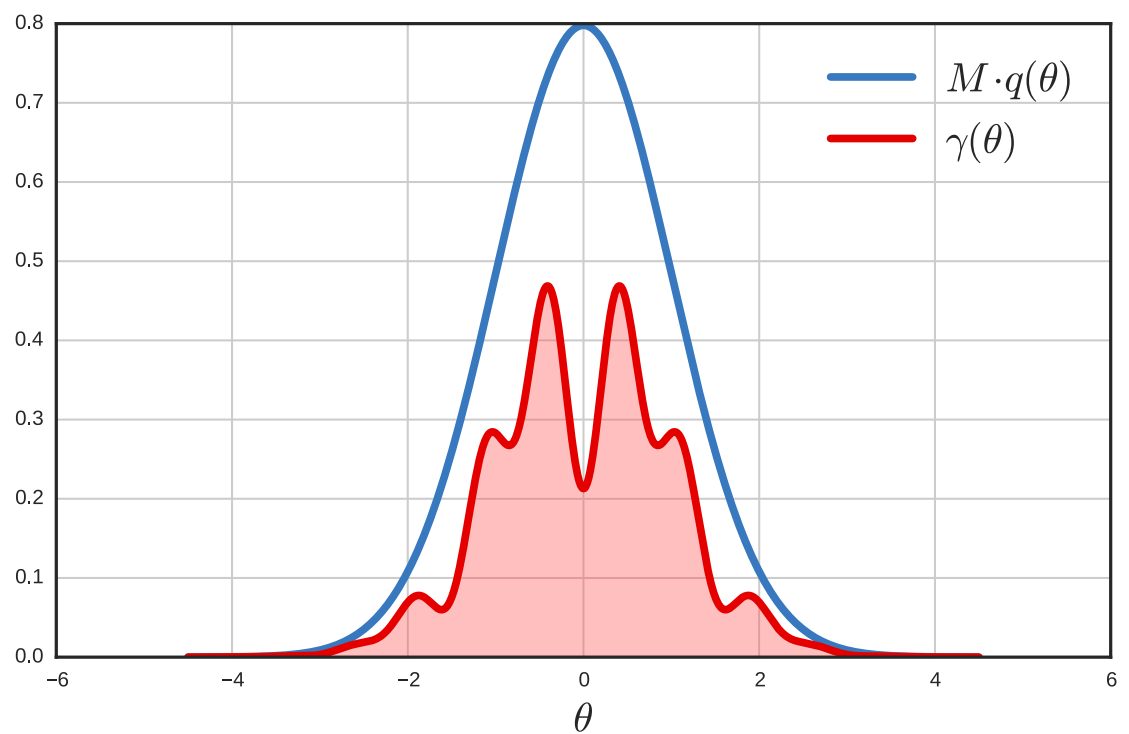

Figure 3.1: Illustration of rejection sampling with target $\gamma(\theta) \propto$ $e^{-\frac{1}{2} \theta^{2}}\left(1+\sin ^{2}(4 \theta)+3 \cos ^{2}(\theta) \sin ^{2}(\theta)\right)$, proposal $q(\theta)=\mathcal{N}(\theta \mid 0,1)$, and constant $M=2$.

One of the main limitations of rejection sampling is the requirement to find the constant $M$ and proposal $q(\theta)$ that satisfies the constraints and results in few rejected samples. The computational complexity of rejection sampling tends to scale poorly with the dimension of $\theta$. For these reasons, improving on rejection sampling is key to more efficient approximate inference.

\subsection{Variational inference}

Variational methods, also known as variational Bayes, turn the problem of integrating over the parameters (to find the marginal likelihood) into an optimization problem. The solution to the optimization problem results in a simpler distribution that we can efficiently work with. This distribution is chosen so that the discrepancy between it and the posterior is as small as possible. We trace the beginnings of variational methods in machine learning back to at least the early works by Peterson and Anderson (1987) and Hinton and van Camp (1993). This together with insight from Parisi (1988) led to a flurry of work in the area (Jordan et al., 1999; Waterhouse et al., 1996).

We will in this section describe the fundamentals of variational methods for approximating the posterior distribution. Variational inference, together with connections to Monte Carlo methods, are studied in Papers G and H. 


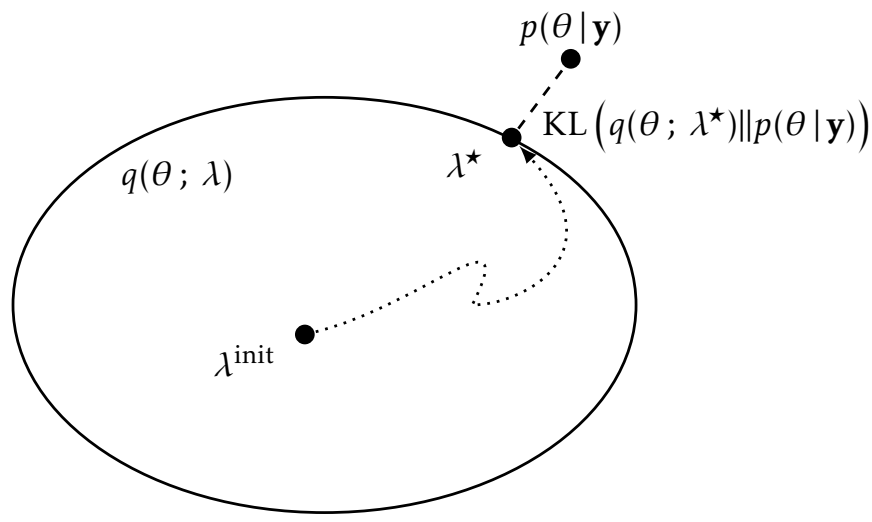

Figure 3.2: Conceptual illustration of variational methods. Each point corresponds to a distribution on $\theta$. The ellipse contains the approximating family $q(\theta ; \lambda)$ indexed by variational parameters $\lambda$.

\subsubsection{The variational idea}

Just like in MC methods, with variational methods we are interested in approximating the posterior distribution. We do this by postulating a variational family of approximations, $q(\theta ; \lambda)$ indexed by the variational parameters $\lambda$. A common example is to use a normal distribution, $q(\theta ; \lambda)=\mathcal{N}\left(\theta \mid \mu, \sigma^{2}\right)$ where $\lambda=(\mu, \sigma)$. The key idea is then to turn to mathematical optimization, choosing $\lambda$ such that we minimize a cost function representing the discrepancy between the variational approximation and the posterior distribution. A common choice of cost function is the Kullback-Leibler (KL) divergence from the variational approximation to the posterior,

$$
\mathrm{KL}(q(\theta ; \lambda) \| p(\theta \mid \mathbf{y}))=\mathbb{E}_{q(\theta ; \lambda)}[\log q(\theta ; \lambda)-\log p(\theta \mid \mathbf{y})] .
$$

For an illustration of generic variational methods for approximate inference see Figure 3.2. However, this expression still requires us to evaluate the posterior distribution, the problem we are trying to solve in the first place.

To resolve the issue we note that minimizing the $\mathrm{KL}$ divergence is equivalent to maximizing the evidence lower bound (ELBO) (Jordan et al., 1999),

$$
\mathrm{L}(\lambda):=\mathbb{E}_{q(\theta ; \lambda)}[\log p(\mathbf{y}, \theta)-\log q(\theta ; \lambda)]
$$

This is true because we can rewrite the KL divergence as follows

$$
\mathrm{KL}(q(\theta ; \lambda) \mid p(\theta \mid \mathbf{y}))=\log p(\mathbf{y})-\mathbb{E}_{q(\theta ; \lambda)}[\log p(\mathbf{y}, \theta)-\log q(\theta ; \lambda)]
$$

Because $\log p(\mathbf{y})$ does not depend on the variational parameters $\lambda$, we can minimize the KL by minimizing the second expression (negative ELBO) on the right hand side. 
The rest of this section will be focused on various ways for maximizing the ELBO, focusing on an explicit coordinate ascent algorithm for mean field approximations and stochastic gradient methods for generic variational approximating families.

\subsubsection{Coordinate ascent variational inference}

Coordinate ascent variational inference (CAVI) is a method for finding an optimal variational approximation when we restrict our family to be independent over the components of $\theta$, i.e.

$$
q(\theta)=\prod_{k=1}^{K} q_{k}\left(\theta_{k}\right)
$$

where we assume $\theta=\left(\theta_{1}, \ldots, \theta_{K}\right)^{\top}$. Note that we have not made any parametric assumptions on the factors $q_{k}(\cdot)$, except that they are probability distributions. This variational family of approximations is known as the mean field variational family.

Under the mean field assumption it is possible to design a coordinate ascent method to optimize the ELBO in Equation (3.13). We update each factor $q_{k}(\cdot)$ one by one, keeping the other $K-1$ factors fixed. A necessary condition in optimization for a point to be optimal is that the derivative is equal to zero. However, in our setting each factor $q_{k}(\cdot)$ is a functional and we instead rely on calculus of variations and functional derivatives (Forsyth, 1960). To ensure that $q_{k}(\cdot)$ is a probability density function we can study the Lagrangian (Bertsekas, 2016; Boyd and Vandenberghe, 2004) $\widetilde{L}$ for Equation (3.13),

$$
\begin{aligned}
& \widetilde{\mathrm{L}}\left(q_{1: K}, v_{1: K}\right)=\mathbb{E}_{\prod_{k} q_{k}\left(\theta_{k}\right)}\left[\log p\left(\mathbf{y}, \theta_{1: K}\right)-\sum_{k=1}^{K} \log q_{k}\left(\theta_{k}\right)\right]-\sum_{k=1}^{K} v_{k}\left(\int q_{k}\left(\theta_{k}\right) \mathrm{d} \theta_{k}-1\right) \\
& =\mathbb{E}_{q_{k}\left(\theta_{k}\right)}\left[\mathbb{E}_{\prod_{m \neq k} q_{m}\left(\theta_{m}\right)}\left[\log p\left(\mathbf{y}, \theta_{1: K}\right)\right]-\log q_{k}\left(\theta_{k}\right)-v_{k}\right]+\text { const., }
\end{aligned}
$$

where $\theta_{1: K}=\left(\theta_{1}, \ldots, \theta_{K}\right)^{\top}=\theta$, the $v_{k}$ 's are the Lagrange multipliers, and const. includes all terms constant with respect to $q_{k}\left(\theta_{k}\right)$. Using the Euler-Lagrange equation we can compute the functional derivative with respect to $q_{k}\left(\theta_{k}\right)$

$$
\frac{\partial \widetilde{\mathrm{L}}\left(q_{1: K}, v_{1: k}\right)}{\partial q_{k}\left(\theta_{k}\right)}=\mathbb{E}_{\prod_{m \neq k} q_{m}\left(\theta_{m}\right)}\left[\log p\left(\mathbf{y}, \theta_{1: K}\right)\right]-\log q_{k}\left(\theta_{k}\right)+\text { const. }
$$

Setting the right-hand side equal to zero gives us the solution for factor $q_{k}^{\star}\left(\theta_{k}\right)$,

$$
q_{k}^{\star}\left(\theta_{k}\right) \propto \exp \left(\mathbb{E}_{\prod_{m \neq k} q_{m}^{\star}\left(\theta_{m}\right)}\left[\log p\left(\mathbf{y}, \theta_{1: K}\right)\right]\right) .
$$

This requires us to be able to evaluate the expectation, and re-normalize the right-hand side of Equation (3.18) with respect to $\theta_{k}$. This is possible when 
the complete conditional $p\left(\theta_{k} \mid \mathbf{y}, \theta_{1}, \ldots, \theta_{k-1}, \theta_{k+1}, \ldots, \theta_{K}\right)$ is in a specific class of exponential family distributions (Blei et al., 2017). For more information on CAVI, and a detailed Bayesian mixture example, see e.g. Bishop (2006); Blei et al. (2017).

CAVI is not applicable if we can not evaluate the expectation and the normalization constant in Equation (3.18). This might be true if our model $p(\mathbf{y}, \theta)$ is too complex. The mean field approximation also makes a strong assumption on the independence between components of the parameter $\theta$. When either of these two assumptions does not hold, we need to resort to a different approach to optimize the ELBO. In the next section, we discuss applying stochastic gradient methods for optimization.

\subsubsection{Stochastic gradient variational inference}

We return to the case when we have a variational family of approximations parameterized by $\lambda$, i.e. $q(\theta ; \lambda)$. When either or both of the model $p(\mathbf{y}, \theta)$ and the variational approximation $q(\theta ; \lambda)$ do not satisfy the requirements for CAVI, we must look to other methods for optimizing the ELBO. A recent approach that has garnered a considerable amount of success is to make use of stochastic gradients to optimize the ELBO (Kingma and Welling, 2014; Mnih and Gregor, 2014; Paisley et al., 2012; Ranganath et al., 2014; Salimans and Knowles, 2013). Essentially we use standard gradient descent with decreasing step-size, but instead of exact gradients we use gradients approximated via MC methods. Stochastic gradient descent is an iterative method for $\lambda$. The update is

$$
\lambda^{n}=\lambda^{n-1}+\alpha_{n} \widehat{\mathrm{g}}\left(\lambda^{n-1}\right),
$$

where $\widehat{\mathrm{g}}\left(\lambda^{n-1}\right)$ is a MC estimator of the ELBO gradient

$$
\mathrm{g}\left(\lambda^{n-1}\right):=\nabla_{\lambda} \mathrm{L}\left(\lambda^{n-1}\right)
$$

and the stepsizes satisfy $\alpha_{n} \geq 0, \sum_{n} \alpha_{n}=\infty, \sum_{n} \alpha_{n}^{2}<\infty$ (Robbins and Monro, 1951).

We review below two of the most common estimators of the ELBO gradient: the score function estimator and the reparameterization estimator.

Score function estimator The score function gradient estimator is based on a reformulation of the ELBO gradient (Mnih and Gregor, 2014; Paisley et al., 2012; Ranganath et al., 2014) as an expectation with respect to the variational approximation $q(\theta ; \lambda)$. The estimator is also known by its other names: the log-derivative trick or REINFORCE (Glynn, 1990; Williams, 1992). It is a generic estimator with very few assumptions on the parameter space or the variational family. It works 
whether $\theta$ is continuous or discrete. We have

$$
\begin{aligned}
\nabla_{\lambda} \mathrm{L}(\lambda) & =\nabla_{\lambda} \mathbb{E}_{q(\theta ; \lambda)}[\log p(\mathbf{y}, \theta)-\log q(\theta ; \lambda)] \\
& =\mathbb{E}_{q(\theta ; \lambda)}\left[(\log p(\mathbf{y}, \theta)-\log q(\theta ; \lambda)) \cdot \nabla_{\lambda} \log q(\theta ; \lambda)\right],
\end{aligned}
$$

where we have made use of the fact that the expectation of the score function $\nabla_{\lambda} \log q(\theta ; \lambda)$ is zero, i.e.

$$
\mathbb{E}_{q(\theta ; \lambda)}\left[\nabla_{\lambda} \log q(\theta ; \lambda)\right]=0,
$$

and the log-derivative trick $\nabla_{\lambda} q(\theta ; \lambda)=q(\theta ; \lambda) \nabla_{\lambda} \log q(\theta ; \lambda)$.

Equation (3.21) suggests estimating the ELBO gradient using the standard MC idea,

$$
\nabla_{\lambda} \mathrm{L}(\lambda) \approx \widehat{\mathrm{g}}_{\text {score }}=\frac{1}{N} \sum_{i=1}^{N}\left(\log p\left(\mathbf{y}, \theta^{i}\right)-\log q\left(\theta^{i} ; \lambda\right)\right) \cdot \nabla_{\lambda} \log q\left(\theta^{i} ; \lambda\right),
$$

where the samples are iid $\theta^{i} \sim q(\cdot ; \lambda)$, just like we discussed in Section 3.1. From the theoretical results of standard MC we know that this estimator is unbiased and consistent.

The main limitation of the score function estimator is that it tends to give quite high variance gradient estimators. This is generally undesirable for an efficient optimization method. It can be partially alleviated by various variance reducing adjustments, such as control variates (Robert and Casella, 2004) and RaoBlackwellization (Casella and Robert, 1996) as explained by e.g. Ranganath et al. (2014).

Reparameterization estimator The reparameterization trick (Bonnet, 1964; Kingma and Welling, 2014; Price, 1958; Salimans and Knowles, 2013) usually results in a gradient estimator with lower variance than the score function estimator. This comes at the price of applicability, where the reparameterization trick only works for a certain class of continuous distributions. We require that the model $p(\mathbf{y}, \theta)$ is differentiable with respect to the parameters $\theta$. Furthermore, we require that the variational approximation $q(\theta ; \lambda)$ is differentiable with respect to $\theta$, and that it is reparameterizable by a differentiable function $\mathrm{f}$. What this means is that we assume that we can simulate from the variational approximation through a noncentered parameterization (Papaspiliopoulos et al., 2003), i.e.

$$
\theta \sim q(\theta ; \lambda) \Longleftrightarrow \theta=\mathrm{f}(\varepsilon, \lambda), \quad \varepsilon \sim p(\varepsilon),
$$

where $\mathrm{f}$ is differentiable with respect to $\lambda$, and the distribution $p(\varepsilon)$ does not depend on the variational parameters $\lambda$. A commonly used variational family is the normal distribution $q(\theta ; \lambda)=\mathcal{N}\left(\theta \mid \mu, \sigma^{2}\right)$, with $\lambda=(\mu, \sigma)$, which has the following non-centered parameterization

$$
\mathrm{f}(\varepsilon, \lambda)=\mu+\sigma \varepsilon, \quad \varepsilon \sim \mathcal{N}(0,1) .
$$


With the non-centered parameterization we can rewrite the gradient of the ELBO using the reparameterization trick

$$
\begin{aligned}
\nabla_{\lambda} \mathrm{L}(\lambda) & =\nabla_{\lambda} \mathbb{E}_{q(\theta ; \lambda)}[\log p(\mathbf{y}, \theta)-\log q(\theta ; \lambda)] \\
& =\nabla_{\lambda} \mathbb{E}_{p(\varepsilon)}[\log p(\mathbf{y}, \mathrm{f}(\varepsilon, \lambda))-\log q(\mathrm{f}(\varepsilon, \lambda) ; \lambda)] \\
& =\mathbb{E}_{p(\varepsilon)}\left[\left.\nabla_{\theta}(\log p(\mathbf{y}, \theta)-\log q(\theta ; \lambda))\right|_{\theta=\mathrm{f}(\varepsilon, \lambda)} \nabla_{\lambda} \mathrm{f}(\varepsilon, \lambda)\right]
\end{aligned}
$$

where in the last equation we have again made use of that the expectation of the score function is zero (Roeder et al., 2017). Equation (3.25) suggests the following $\mathrm{MC}$ estimator of the ELBO gradient

$$
\nabla_{\lambda} \mathrm{L}(\lambda) \approx \widehat{\mathrm{g}}_{\text {reparam }}=\left.\frac{1}{N} \sum_{i=1}^{N} \nabla_{\theta}(\log p(\mathbf{y}, \theta)-\log q(\theta ; \lambda))\right|_{\theta=\mathrm{f}\left(\varepsilon^{i}, \lambda\right)} \nabla_{\lambda} \mathrm{f}\left(\varepsilon^{i}, \lambda\right),
$$

where the samples are iid $\varepsilon^{i} \sim p(\varepsilon)$. In practice, the variance of the reparameterization estimator is often low enough that a single sample, $N=1$, is sufficient (Kingma and Welling, 2014; Roeder et al., 2017).

The restriction that the variational approximation must be reparameterizable can be alleviated using partial reparameterization, see e.g. Ruiz et al. (2016) and Paper G.

Large-scale data Datasets where the number of (exchangeable) datapoints $T$ is very large works well in the setting of stochastic gradient-based variational inference. We simply replace evaluating the exact $\log$-likelihood $\log p(\mathbf{y} \mid \theta)$ with an unbiased estimate of it, i.e.

$$
\log p(\mathbf{y} \mid \theta)=\sum_{t=1}^{T} \log p\left(y_{t} \mid \theta\right) \approx \frac{T}{B} \sum_{b=1}^{B} \log p\left(y_{\tau_{b}} \mid \theta\right), \quad \tau_{b} \sim \mathrm{U}(\{1, \ldots, T\}) .
$$

Here $\tau_{b}$ are discrete random variables drawn uniformly over the support $\{1, \ldots, T\}$. When used together with either of the two stochastic gradient methods above, the resulting algorithm is known as a doubly stochastic algorithm (Titsias and Lázaro-Gredilla, 2014).

\subsubsection{Variational expectation-maximization}

The expectation-maximization (EM) algorithm (Dempster et al., 1977) is a method for finding the maximum likelihood estimate $\widehat{\theta}^{\mathrm{ML}}$ when the model depends on local latent (unobserved) variables $\mathbf{x}$. This means that our probabilistic model is $p(\mathbf{y}, \mathbf{x} \mid \theta)$, and we are interested in maximizing the marginal likelihood $p(\mathbf{y} \mid \theta)=$ 
$\int p(\mathbf{y}, \mathbf{x} \mid \theta) \mathrm{d} \mathbf{x}$ with respect to the parameters $\theta$. The EM algorithm solves this through coordinate ascent for the ELBO defined by

$$
\mathrm{L}(q(\mathbf{x}), \theta)=\mathbb{E}_{q(\mathbf{x})}[\log p(\mathbf{y}, \mathbf{x} \mid \theta)-\log q(\mathbf{x})],
$$

where $q(\mathbf{x})$ is a distribution over $\mathbf{x}$. The EM algorithm consists of iteratively solving

$$
\begin{aligned}
q^{n}(\mathbf{x}) & =\underset{q(\mathbf{x})}{\arg \max } \mathrm{L}\left(q(\mathbf{x}), \theta^{n-1}\right), \\
\theta^{n} & =\underset{\theta}{\arg \max } \mathrm{L}\left(q^{n}(\mathbf{x}), \theta\right),
\end{aligned}
$$

where the solution to the first line is given by $q^{n}(\mathbf{x})=p\left(\mathbf{x} \mid \mathbf{y}, \theta^{n-1}\right)$.

One of the first approaches to variational expectation-maximization (VEM) (Beal and Ghahramani, 2003) ${ }^{1}$ replaces Equation (3.29a) (maximization for $q(\mathbf{x})$ ), by CAVI. While the exact EM (under suitable conditions) is guaranteed to converge to a local maxima of $\log p(\mathbf{y} \mid \theta)$, this is generally not true for VEM. Just like variational inference and MC methods are approximate methods for inference, so is VEM.

Because VEM relies on CAVI, it has restricted applicability. Recent research has focused on using stochastic gradient-based variational inference instead. In this setting we iteratively do only partial updates for $q$ and $\theta$, unlike in Equation (3.29). We let the variational approximation be defined by $q(\mathbf{x} ; \lambda)$, and design an update scheme for $\lambda, \theta$ based on stochastic gradient descent for the ELBO

$$
\mathrm{L}(\lambda, \theta)=\mathbb{E}_{q(\mathbf{x} ; \lambda)}[\log p(\mathbf{y}, \mathbf{x} \mid \theta)-\log q(\mathbf{x} ; \lambda)] .
$$

Based on step-size sequences $\alpha_{n}^{\lambda}, \alpha_{n}^{\theta}$ (satisfying the same requirements as detailed in Section 3.2.3), we get

$$
\begin{aligned}
& \lambda^{n}=\lambda^{n-1}+\alpha_{n}^{\lambda} \hat{\mathrm{g}}_{n}^{\lambda}, \\
& \theta^{n}=\theta^{n-1}+\alpha_{n}^{\theta} \hat{\mathrm{g}}_{n}^{\theta},
\end{aligned}
$$

where $\hat{\mathrm{g}}_{n}^{\theta}$ is

$$
\hat{\mathrm{g}}_{n}^{\theta}=\left.\frac{1}{N} \sum_{i=1}^{N} \nabla_{\theta} \log p\left(\mathbf{y}, \mathbf{x}^{i} \mid \theta\right)\right|_{\theta=\theta^{n-1}}, \quad \mathbf{x}^{i} \sim q\left(\mathbf{x} ; \lambda^{n-1}\right) .
$$

For the gradient with respect to $\lambda, \hat{\mathrm{g}}_{n}^{\lambda}$, we make use of either the score function or reparameterization trick estimators of Section 3.2.3, evaluated for $\lambda^{n-1}$, $\theta^{n-1}$. Because the algorithm we described is a form of stochastic gradient descentalgorithm, it will (under suitable conditions) converge to a local maxima of the evidence lower bound in Equation (3.30) (Bottou et al., 2018). However, there are no guarantees for the original problem of maximum marginal likelihood maximization. We are maximizing a lower bound to the marginal likelihood, where the gap between them that we neglect is a function of both $\lambda$ and $\theta$.

\footnotetext{
${ }^{1}$ The authors actually consider model selection: $p(\mathbf{y}, \mathbf{x}, \theta \mid m)$ and the mean-field model $q(\theta) q(\mathbf{x})$ where $m$ is a model selection variable. The fundamental idea is the same.
} 



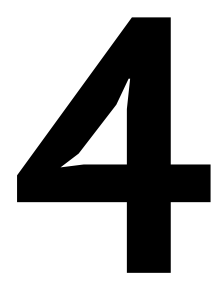

\section{Concluding remarks}

We conclude Part I of this thesis by a short summary, and discuss potential avenues for future work in approximate inference. However, we note that more discussion is also provided at the end of most articles in Part II.

In this thesis we introduce various new methods for approximate inference and learning in latent variable models. The focus has been on sequential Monte Carlo and variational inference, as well as the connection between them. At the heart of each paper lies MC methods, used to estimate intractable integrals. We present a tutorial on, new applications for, and extensions to sequential Monte Carlo. We also study reparameterization gradients for variational inference (VI), and derive the variational sequential Monte Carlo (VSMC) family of approximations to the posterior.

We would like to take the opportunity to focus the discussion on nested Monte Carlo and variational Monte Carlo, two open avenues for future research.

Nested Monte Carlo We refer to the use of MC methods within other MC methods as nested Monte Carlo (NMC) algorithms. Examples include, but are not limited to,

- Markov chain Monte Carlo within SMC, e.g. sequential Monte Carlo samplers (Del Moral et al., 2006),

- SMC within Markov chain Monte Carlo, e.g. particle Markov chain Monte Carlo (Andrieu et al., 2010),

- SMC within SMC, e.g. nested sequential Monte Carlo (Papers D, E) or SMC ${ }^{2}$ (Chopin et al., 2012). 
This area is by no means exhausted, particularly the intersection between different NMC algorithms. We believe that nested sequential Monte Carlo (NSMC) is potentially useful for a much wider range of applications than it has been applied to so far. More generally, how can we design NMC algorithms such that we preserve the theoretical guarantees? Can we automatically choose the right algorithm for a particular task? Is it possible to give practical guarantees on the approximation we obtain?

Variational Monte Carlo By synthesizing approximate inference from both variational and MC methods, we get variational Monte Carlo (VMC). We can also think of this as a form of adaptive MC method. Examples include, but are not limited to,

- Markov chain Monte Carlo-inspired approximations, e.g. Hamiltonian variational inference (Salimans et al., 2015),

- importance sampling within variational inference, e.g. variational importance sampling (Paper H), (Cremer et al., 2017),

- SMC within variational inference, e.g. variational sequential Monte Carlo (Paper H).

Explicitly making use of the MC approximation of the posterior when defining a variational approximation is a largely unexplored area. Can we efficiently learn the corresponding variational parameters? Will investigating different cost functions help improve the approximation? Is it possible to extract informative theoretical guarantees for this class of approximations? How can we better take into account the discrete nature, introduced by the use of MC, of the approximations?

The above two examples are areas that can potentially have a significant impact on approximate inference for probabilistic machine learning. 


\section{Bibliography}

Christophe Andrieu, Arnaud Doucet, and Roman Holenstein. Particle Markov chain Monte Carlo methods. Journal of the Royal Statistical Society: Series B (Statistical Methodology), 72(3):269-342, 2010.

Thomas Bayes. An essay towards solving a problem in the doctrine of chances. Philosophical Transactions of the Royal Society, pages 330-418, 1763.

Matthew J. Beal and Zoubin Ghahramani. The variational Bayesian EM algorithm for incomplete data: with application to scoring graphical model structures. Bayesian statistics, 2003.

Dimitri P. Bertsekas, editor. Nonlinear Programming. Athena Scientific, 2016.

Christopher M. Bishop. Pattern Recognition and Machine Learning. Springer Science+Business Media, 2006.

David M. Blei, Alp Kucukelbir, and Jon D. McAuliffe. Variational inference: A review for statisticians. Journal of the American Statistical Association, 2017.

G. Bonnet. Transformations des signaux aléatoires a travers les systemes non linéaires sans mémoire. Annals of Telecommunications, 19(9):203-220, 1964.

L. Bottou, F. Curtis, and J. Nocedal. Optimization methods for large-scale machine learning. SIAM Review, 60(2):223-311, 2018.

Stephen Boyd and Lieven Vandenberghe. Convex optimization. Cambridge university press, 2004.

Olivier Cappé, Eric Moulines, and Tobias Ryden. Inference in Hidden Markov Models. Springer Science+Business Media, 2005.

G. Casella and C. P. Robert. Rao-Blackwellisation of sampling schemes. Biometrika, 83(1):81-94, 1996.

George Casella and Roger L Berger. Statistical inference. Duxbury, 2002. 
N. Chopin, P. E. Jacob, and O. Papaspiliopoulos. Smc2: an efficient algorithm for sequential analysis of state space models. Journal of the Royal Statistical Society: Series B (Statistical Methodology), 75(3):397-426, 2012.

C. Cremer, Q. Morris, and D. Duvenaud. Reinterpreting importance-weighted autoencoders. arXiv:1704.02916, April 2017.

P. Del Moral, A. Doucet, and A. Jasra. Sequential Monte Carlo samplers. Journal of the Royal Statistical Society: Series B, 68(3):411-436, 2006.

A. P. Dempster, N. M. Laird, and D. B. Rubin. Maximum likelihood from incomplete data via the em algorithm. Journal of the Royal Statistical Society. Series B (Methodological), 39(1):1-38, 1977.

Luc Devroye. Non-Uniform Random Variate Generation. Springer-Verlag, 1986.

Roger Eckhardt. Stan Ulam, John von Neumann, and the Monte Carlo method. Los Alamos Science, 15(131-136):30, 1987.

Bradley Efron and Trevor Hastie, editors. Computer Age Statistical Inference: Algorithms, Evidence, and Data Science. Cambridge University Press, 2016.

Ronald A. Fisher. On the mathematical foundations of theoretical statistics. Philosophical Transactions of the Royal Society of London A: Mathematical, Physical and Engineering Sciences, 222(594-604):309-368, 1922.

A. S. Forsyth. Calculus of variations. Dover Publications Inc., 1960.

Jerome Friedman, Trevor Hastie, and Robert Tibshirani. The elements of statistical learning. Springer Science+Business Media, 2009.

Andrew Gelman, Hal S Stern, John B Carlin, David B Dunson, Aki Vehtari, and Donald B Rubin. Bayesian data analysis. Chapman and Hall/CRC, 2013.

Zoubin Ghahramani. Probabilistic machine learning and artificial intelligence. Nature, 2015.

P. W. Glynn. Likelihood ratio gradient estimation for stochastic systems. Communications of the ACM, 33(10):75-84, oct 1990.

Anders Hald. On the history of maximum likelihood in relation to inverse probability and least squares. Statistical Science, 14(2):214-222, 1999.

Geoffrey E. Hinton and Drew van Camp. Keeping the neural networks simple by minimizing the description length of the weights. In Proceedings of the Sixth Annual Conference on Computational Learning Theory, pages 5-13, New York, NY, USA, 1993. ACM.

M. I. Jordan, Z. Ghahramani, T. S. Jaakkola, and L. K. Saul. An introduction to variational methods for graphical models. Machine Learning, 37(2):183-233, November 1999. 
D. P. Kingma and M. Welling. Auto-encoding variational Bayes. In International Conference on Learning Representations, 2014.

Daphne Koller, Nir Friedman, and Francis Bach. Probabilistic graphical models: principles and techniques. MIT press, 2009.

Pierre-Simon Laplace. Mémoire sur la probabilité des causes par led évènemens. Mémoires de Mathématque et Physique, Presentés à l'Académie Royale des Sciences, par divers Savans \& lûs dans ses Assemblées, Tome Sixiéme, 66:621-56, 1774 .

Fredrik Lindsten, Adam M. Johansen, Christian A. Naesseth, Brent Kirkpatrick, Thomas B. Schön, John Aston, and Alexandre Bouchard-Côté. Divide-andconquer with sequential Monte Carlo. Journal of Computational and Graphical Statistics, 26(2):445-458, 2017.

Jun S Liu. Monte Carlo strategies in scientific computing. Springer Science+Business Media, 2004

Nicholas Metropolis and Stanislaw Ulam. The Monte Carlo method. Journal of the American statistical association, 44(247):335-341, 1949.

A. Mnih and K. Gregor. Neural variational inference and learning in belief networks. In International Conference on Machine Learning, 2014.

Christian Naesseth, Fredrik Lindsten, and Thomas Schön. Nested sequential Monte Carlo methods. In International Conference on Machine Learning (ICML), pages 1292-1301, 2015a.

Christian A. Naesseth, Fredrik Lindsten, and Thomas B. Schön. Capacity estimation of two-dimensional channels using sequential Monte Carlo. In IEEE Information Theory Workshop (ITW), pages 431-435, $2014 \mathrm{a}$.

Christian A. Naesseth, Fredrik Lindsten, and Thomas B. Schön. Sequential Monte Carlo for graphical models. In Advances in Neural Information Processing Systems (NIPS), pages 1862-1870, 2014b.

Christian A. Naesseth, Fredrik Lindsten, and Thomas B. Schön. Towards automated sequential Monte Carlo for probabilistic graphical models. In NIPS Workshop on Black Box Learning and Inference, $2015 \mathrm{~b}$.

Christian A. Naesseth, Fredrik Lindsten, and Thomas B. Schön. Highdimensional filtering using nested sequential Monte Carlo. arXiv:1612.09162, 2016.

Christian A. Naesseth, Francisco Ruiz, Scott W. Linderman, and David M. Blei. Reparameterization gradients through acceptance-rejection sampling algorithms. In Artificial Intelligence and Statistics (AISTATS), pages 489-498, 2017. 
Christian A. Naesseth, Scott W. Linderman, Rajesh Ranganath, and David M. Blei. Variational sequential Monte Carlo. In Artificial Intelligence and Statistics (AISTATS), pages 968-977, 2018a.

Christian A. Naesseth, Fredrik Lindsten, and Thomas B. Schön. Elements of sequential Monte Carlo. Foundations and Trends in Machine Learning, 2018b. (proposal accepted, manuscript in preparation).

Jorge Nocedal and S. Wright, editors. Numerical Optimization. Springer Science+Business Media, 2006.

J. W. Paisley, D. M. Blei, and M. I. Jordan. Variational Bayesian inference with stochastic search. In International Conference on Machine Learning, 2012.

Sina Khoshfetrat Pakazad, Christian A. Naesseth, Fredrik Lindsten, and Anders Hansson. Distributed, scalable and gossip-free consensus optimization with application to data analysis. arXiv:1705.02469, 2017.

Omiros Papaspiliopoulos, Gareth O. Roberts, and Martin Sköld. Non-centered parameterisations for hierarchical models and data augmentation. In Bayesian Statistics 7: Proceedings of the Seventh Valencia International Meeting, page 307. Oxford University Press, USA, 2003.

Giorgio Parisi. Statistical field theory. Addison-Wesley, 1988.

Carsten Peterson and James R. Anderson. A mean field theory learning algorithm for neural networks. Complex Systems, 1(5), 1987.

R. Price. A useful theorem for nonlinear devices having Gaussian inputs. IRE Transactions on Information Theory, 4(2):69-72, 1958.

Tom Rainforth, Christian A. Naesseth, Fredrik Lindsten, Brooks Paige, JanWillem Vandemeent, Arnaud Doucet, and Frank Wood. Interacting particle Markov chain Monte Carlo. In International Conference on Machine Learning (ICML), pages 2616-2625, 2016.

Rajesh Ranganath, Sean Gerrish, and David M. Blei. Black box variational inference. In Artificial Intelligence and Statistics, 2014.

Herbert Robbins and Sutton Monro. A stochastic approximation method. The Annals of Mathematical Statistics, 22(3):400-407, 091951.

Christian P. Robert. The Bayesian Choice. Springer Science+Business Media, 2007.

Christian P. Robert and George Casella. Monte Carlo statistical methods. Springer Science+Business Media, 2004.

Geoffrey Roeder, Yuhuai Wu, and David K Duvenaud. Sticking the landing: Simple, lower-variance gradient estimators for variational inference. In Advances in Neural Information Processing Systems, pages 6925-6934, 2017. 
Francisco J. R. Ruiz, Michalis K. Titsias, and David M. Blei. The generalized reparameterization gradient. In Advances in Neural Information Processing Systems, 2016.

Tim Salimans and David A. Knowles. Fixed-form variational posterior approximation through stochastic linear regression. Bayesian Analysis, 8(4):837-882, 2013.

Tim Salimans, Diederik P. Kingma, and Max Welling. Markov chain Monte Carlo and variational inference: Bridging the gap. In International Conference on Machine Learning, 2015.

Thomas B. Schön, Fredrik Lindsten, Johan Dahlin, Johan Wågberg, Christian A. Naesseth, Andreas Svensson, and Liang Dai. Sequential Monte Carlo methods for system identification. IFAC-PapersOnLine (SYSID), 48(28):775-786, 2015.

Stephen M Stigler. The history of statistics: The measurement of uncertainty before 1900. Harvard University Press, 1986.

M. K. Titsias and M. Lázaro-Gredilla. Doubly stochastic variational Bayes for non-conjugate inference. In International Conference on Machine Learning, 2014.

David A Van Dyk and Xiao-Li Meng. The art of data augmentation. Journal of Computational and Graphical Statistics, 10(1):1-50, 2001.

John von Neumann. Various Techniques Used in Connection with Random Digits. Journal of Research of the National Bureau of Standards, 12:36-38, 1951.

Steve Waterhouse, David Mackay, and Tony Robinson. Bayesian methods for mixtures of experts. In Advances in Neural Information Processing Systems, pages 351-357. MIT Press, 1996.

R. J. Williams. Simple statistical gradient-following algorithms for connectionist reinforcement learning. Machine Learning, 8(3-4):229-256, 1992. 



\section{Part II}

\section{Publications}





\section{Publications}

The publications associated with this thesis have been removed for copyright reasons. For more details about these see:

http://urn.kb.se/resolve?urn=urn:nbn:se:liu:diva-152647 


\section{PhD Dissertations \\ Division of Automatic Control \\ Linköping University}

M. Millnert: Identification and control of systems subject to abrupt changes. Thesis No. 82, 1982. ISBN 91-7372-542-0.

A. J. M. van Overbeek: On-line structure selection for the identification of multivariable systems. Thesis No. 86, 1982. ISBN 91-7372-586-2.

B. Bengtsson: On some control problems for queues. Thesis No. 87, 1982. ISBN 91-7372593-5.

S. Ljung: Fast algorithms for integral equations and least squares identification problems. Thesis No. 93, 1983. ISBN 91-7372-641-9.

H. Jonson: A Newton method for solving non-linear optimal control problems with general constraints. Thesis No. 104, 1983. ISBN 91-7372-718-0.

E. Trulsson: Adaptive control based on explicit criterion minimization. Thesis No. 106, 1983. ISBN 91-7372-728-8.

K. Nordström: Uncertainty, robustness and sensitivity reduction in the design of single input control systems. Thesis No. 162, 1987. ISBN 91-7870-170-8.

B. Wahlberg: On the identification and approximation of linear systems. Thesis No. 163, 1987. ISBN 91-7870-175-9.

S. Gunnarsson: Frequency domain aspects of modeling and control in adaptive systems. Thesis No. 194, 1988. ISBN 91-7870-380-8.

A. Isaksson: On system identification in one and two dimensions with signal processing applications. Thesis No. 196, 1988. ISBN 91-7870-383-2.

M. Viberg: Subspace fitting concepts in sensor array processing. Thesis No. 217, 1989. ISBN 91-7870-529-0.

K. Forsman: Constructive commutative algebra in nonlinear control theory. Thesis No. 261, 1991. ISBN 91-7870-827-3.

F. Gustafsson: Estimation of discrete parameters in linear systems. Thesis No. 271, 1992. ISBN 91-7870-876-1.

P. Nagy: Tools for knowledge-based signal processing with applications to system identification. Thesis No. 280, 1992. ISBN 91-7870-962-8.

T. Svensson: Mathematical tools and software for analysis and design of nonlinear control systems. Thesis No. 285, 1992. ISBN 91-7870-989-X.

S. Andersson: On dimension reduction in sensor array signal processing. Thesis No. 290, 1992. ISBN 91-7871-015-4.

H. Hjalmarsson: Aspects on incomplete modeling in system identification. Thesis No. 298, 1993. ISBN 91-7871-070-7.

I. Klein: Automatic synthesis of sequential control schemes. Thesis No. 305, 1993. ISBN 91-7871-090-1.

J.-E. Strömberg: A mode switching modelling philosophy. Thesis No. 353, 1994. ISBN 917871-430-3.

K. Wang Chen: Transformation and symbolic calculations in filtering and control. Thesis No. 361, 1994. ISBN 91-7871-467-2.

T. McKelvey: Identification of state-space models from time and frequency data. Thesis No. 380, 1995. ISBN 91-7871-531-8.

J. Sjöberg: Non-linear system identification with neural networks. Thesis No. 381, 1995. ISBN 91-7871-534-2.

R. Germundsson: Symbolic systems - theory, computation and applications. Thesis No. 389, 1995. ISBN 91-7871-578-4. 
P. Pucar: Modeling and segmentation using multiple models. Thesis No. 405, 1995. ISBN 91-7871-627-6.

H. Fortell: Algebraic approaches to normal forms and zero dynamics. Thesis No. 407, 1995. ISBN 91-7871-629-2.

A. Helmersson: Methods for robust gain scheduling. Thesis No. 406, 1995. ISBN 91-7871628-4.

P. Lindskog: Methods, algorithms and tools for system identification based on prior knowledge. Thesis No. 436, 1996. ISBN 91-7871-424-8.

J. Gunnarsson: Symbolic methods and tools for discrete event dynamic systems. Thesis No. 477, 1997. ISBN 91-7871-917-8.

M. Jirstrand: Constructive methods for inequality constraints in control. Thesis No. 527, 1998. ISBN 91-7219-187-2.

U. Forssell: Closed-loop identification: Methods, theory, and applications. Thesis No. 566, 1999. ISBN 91-7219-432-4.

A. Stenman: Model on demand: Algorithms, analysis and applications. Thesis No. 571, 1999. ISBN 91-7219-450-2.

N. Bergman: Recursive Bayesian estimation: Navigation and tracking applications. Thesis No. 579, 1999. ISBN 91-7219-473-1.

K. Edström: Switched bond graphs: Simulation and analysis. Thesis No. 586, 1999. ISBN 91-7219-493-6.

M. Larsson: Behavioral and structural model based approaches to discrete diagnosis. Thesis No. 608, 1999. ISBN 91-7219-615-5.

F. Gunnarsson: Power control in cellular radio systems: Analysis, design and estimation. Thesis No. 623, 2000. ISBN 91-7219-689-0.

V. Einarsson: Model checking methods for mode switching systems. Thesis No. 652, 2000. ISBN 91-7219-836-2.

M. Norrlöf: Iterative learning control: Analysis, design, and experiments. Thesis No. 653, 2000. ISBN 91-7219-837-0.

F. Tjärnström: Variance expressions and model reduction in system identification. Thesis No. 730, 2002. ISBN 91-7373-253-2.

J. Löfberg: Minimax approaches to robust model predictive control. Thesis No. 812, 2003. ISBN 91-7373-622-8.

J. Roll: Local and piecewise affine approaches to system identification. Thesis No. 802, 2003. ISBN 91-7373-608-2.

J. Elbornsson: Analysis, estimation and compensation of mismatch effects in A/D converters. Thesis No. 811, 2003. ISBN 91-7373-621-X.

O. Härkegård: Backstepping and control allocation with applications to flight control. Thesis No. 820, 2003. ISBN 91-7373-647-3.

R. Wallin: Optimization algorithms for system analysis and identification. Thesis No. 919, 2004. ISBN 91-85297-19-4.

D. Lindgren: Projection methods for classification and identification. Thesis No. 915, 2005. ISBN 91-85297-06-2.

R. Karlsson: Particle Filtering for Positioning and Tracking Applications. Thesis No. 924, 2005. ISBN 91-85297-34-8.

J. Jansson: Collision Avoidance Theory with Applications to Automotive Collision Mitigation. Thesis No. 950, 2005. ISBN 91-85299-45-6.

E. Geijer Lundin: Uplink Load in CDMA Cellular Radio Systems. Thesis No. 977, 2005. ISBN 91-85457-49-3.

M. Enqvist: Linear Models of Nonlinear Systems. Thesis No. 985, 2005. ISBN 91-8545764-7.

T. B. Schön: Estimation of Nonlinear Dynamic Systems - Theory and Applications. Thesis No. 998, 2006. ISBN 91-85497-03-7. 
I. Lind: Regressor and Structure Selection - Uses of ANOVA in System Identification. Thesis No. 1012, 2006. ISBN 91-85523-98-4.

J. Gillberg: Frequency Domain Identification of Continuous-Time Systems Reconstruction and Robustness. Thesis No. 1031, 2006. ISBN 91-85523-34-8.

M. Gerdin: Identification and Estimation for Models Described by Differential-Algebraic Equations. Thesis No. 1046, 2006. ISBN 91-85643-87-4.

C. Grönwall: Ground Object Recognition using Laser Radar Data - Geometric Fitting, Performance Analysis, and Applications. Thesis No. 1055, 2006. ISBN 91-85643-53-X.

A. Eidehall: Tracking and threat assessment for automotive collision avoidance. Thesis No. 1066, 2007. ISBN 91-85643-10-6.

F. Eng: Non-Uniform Sampling in Statistical Signal Processing. Thesis No. 1082, 2007. ISBN 978-91-85715-49-7.

E. Wernholt: Multivariable Frequency-Domain Identification of Industrial Robots. Thesis No. 1138, 2007. ISBN 978-91-85895-72-4.

D. Axehill: Integer Quadratic Programming for Control and Communication. Thesis No. 1158, 2008. ISBN 978-91-85523-03-0.

G. Hendeby: Performance and Implementation Aspects of Nonlinear Filtering. Thesis No. 1161, 2008. ISBN 978-91-7393-979-9.

J. Sjöberg: Optimal Control and Model Reduction of Nonlinear DAE Models. Thesis No. 1166, 2008. ISBN 978-91-7393-964-5.

D. Törnqvist: Estimation and Detection with Applications to Navigation. Thesis No. 1216, 2008. ISBN 978-91-7393-785-6.

P-J. Nordlund: Efficient Estimation and Detection Methods for Airborne Applications. Thesis No. 1231, 2008. ISBN 978-91-7393-720-7.

H. Tidefelt: Differential-algebraic equations and matrix-valued singular perturbation. Thesis No. 1292, 2009. ISBN 978-91-7393-479-4.

H. Ohlsson: Regularization for Sparseness and Smoothness - Applications in System Identification and Signal Processing. Thesis No. 1351, 2010. ISBN 978-91-7393-287-5.

S. Moberg: Modeling and Control of Flexible Manipulators. Thesis No. 1349, 2010. ISBN 978-91-7393-289-9.

J. Wallén: Estimation-based iterative learning control. Thesis No. 1358, 2011. ISBN 978 91-7393-255-4.

J. D. Hol: Sensor Fusion and Calibration of Inertial Sensors, Vision, Ultra-Wideband and GPS. Thesis No. 1368, 2011. ISBN 978-91-7393-197-7.

D. Ankelhed: On the Design of Low Order H-infinity Controllers. Thesis No. 1371, 2011. ISBN 978-91-7393-157-1.

C. Lundquist: Sensor Fusion for Automotive Applications. Thesis No. 1409, 2011. ISBN 978-91-7393-023-9.

P. Skoglar: Tracking and Planning for Surveillance Applications. Thesis No. 1432, 2012. ISBN 978-91-7519-941-2.

K. Granström: Extended target tracking using PHD filters. Thesis No. 1476, 2012. ISBN 978-91-7519-796-8.

C. Lyzell: Structural Reformulations in System Identification. Thesis No. 1475, 2012. ISBN 978-91-7519-800-2.

J. Callmer: Autonomous Localization in Unknown Environments. Thesis No. 1520, 2013. ISBN 978-91-7519-620-6.

D. Petersson: A Nonlinear Optimization Approach to H2-Optimal Modeling and Control. Thesis No. 1528, 2013. ISBN 978-91-7519-567-4.

Z. Sjanic: Navigation and Mapping for Aerial Vehicles Based on Inertial and Imaging Sensors. Thesis No. 1533, 2013. ISBN 978-91-7519-553-7. 
F. Lindsten: Particle Filters and Markov Chains for Learning of Dynamical Systems. Thesis No. 1530, 2013. ISBN 978-91-7519-559-9.

P. Axelsson: Sensor Fusion and Control Applied to Industrial Manipulators. Thesis No. 1585, 2014. ISBN 978-91-7519-368-7.

A. Carvalho Bittencourt: Modeling and Diagnosis of Friction and Wear in Industrial Robots. Thesis No. 1617, 2014. ISBN 978-91-7519-251-2.

M. Skoglund: Inertial Navigation and Mapping for Autonomous Vehicles. Thesis No. 1623, 2014. ISBN 978-91-7519-233-8.

S. Khoshfetrat Pakazad: Divide and Conquer: Distributed Optimization and Robustness Analysis. Thesis No. 1676, 2015. ISBN 978-91-7519-050-1.

T. Ardeshiri: Analytical Approximations for Bayesian Inference. Thesis No. 1710, 2015. ISBN 978-91-7685-930-8.

N. Wahlström: Modeling of Magnetic Fields and Extended Objects for Localization Applications. Thesis No. 1723, 2015. ISBN 978-91-7685-903-2.

J. Dahlin: Accelerating Monte Carlo methods for Bayesian inference in dynamical models. Thesis No. 1754, 2016. ISBN 978-91-7685-797-7.

M. Kok: Probabilistic modeling for sensor fusion with inertial measurements. Thesis No. 1814, 2016. ISBN 978-91-7685-621-5.

J. Linder: Indirect System Identification for Unknown Input Problems: With Applications to Ships. Thesis No. 1829, 2017. ISBN 978-91-7685-588-1.

M. Roth: Advanced Kalman Filtering Approaches to Bayesian State Estimation. Thesis No. 1832, 2017. ISBN 978-91-7685-578-2.

I. Nielsen: Structure-Exploiting Numerical Algorithms for Optimal Control. Thesis No. 1848, 2017. ISBN 978-91-7685-528-7.

D. Simon: Fighter Aircraft Maneuver Limiting Using MPC: Theory and Application. Thesis No. 1881, 2017. ISBN 978-91-7685-450-1.

C. Veibäck: Tracking the Wanders of Nature. Thesis No. 1958, 2018. ISBN 978-91-7685200-2. 